\title{
Dark Matter in Elliptical Galaxies
}

David A. Buote and Philip J. Humphrey

\begin{abstract}
We review X-ray constraints on dark matter in giant elliptical galaxies $\left(10^{12} \mathrm{M}_{\odot} \lesssim \mathrm{M}_{\mathrm{vir}} \lesssim 10^{13} \mathrm{M}_{\odot}\right)$ obtained using the current generation of X-ray satellites, beginning with an overview of the physics of the hot interstellar medium and mass modeling methodology. Dark matter is now firmly established in many galaxies, with inferred NFW concentration parameters somewhat larger than the mean theoretical relation. X-ray observations confirm that the total mass profile (baryons+DM) is close to isothermal $(M \sim r)$, and new evidence suggests a more general power-law relation for the slope of the total mass profile that varies with the stellar half-light radius. We also discuss constraints on the baryon fraction, supermassive black holes, and axial ratio of the dark matter halo. Finally, we review constraints on non-thermal gas motions and discuss the accuracy of the hydrostatic equilibrium approximation in elliptical galaxies.
\end{abstract}

\section{Introduction}

Cold, non-baryonic dark matter (CDM) is a critical ingredient of the widely accepted Big Bang cosmological paradigm (1; 2; 3). Although the dark matter (DM) particle has yet to be directly detected in a terrestrial laboratory $(4 ; 5 ;$; 6 ), the total amount of DM is known precisely from observations of the large-scale geometry of the Universe, in particular the cosmic microwave background radiation, distant Type Ia supernovas, baryon acoustic oscillations, galaxy cluster mass functions and baryon fractions. These observations indicate that DM comprises about $23 \%$ of the energy density budget of the Universe today compared to about $4 \%$ for baryonic matter (7; 8).

While the standard cosmological model describes the large-scale Universe very well, it has encountered interesting tension with observations of DM on galaxy

Department of Physics and Astronomy, 4129 Frederick Reines Hall, University of California at Irvine, Irvine, CA 92697-4575, e-mail:buote@uci.edu, phumphre@uci.edu 
scales. Perhaps most notably, beginning with the seminal studies of rotation curves of dwarf spiral galaxies (9; 10), it is now generally recognized that both small and large disk galaxies have central radial DM profiles that are flatter than initially predicted by the standard model when considering only the gravitational influence of the DM $(11 ; 12 ; 13)$. Similar cored DM profiles are obtained by optical studies of elliptical galaxies at the center of galaxy clusters $(14 ; 15 ;$; 16 ; 17; 18), though X-ray observations of some clusters find "cuspy" total mass profiles consistent with theory $(19 ; 20)$. This "core-cusp" problem has generated great interest as it may yield information on the nature of the DM that is apparently inaccessible to observations on larger scales. An influential early suggestion to reconcile observation and theory is that the DM particles are self-interacting $(21 ; 22 ; 23 ; 24)$, though observations apparently do not currently favor that possibility $(19 ; 20 ; 25 ; 26)$.

Alternatively, the core-cusp problem may reveal clues to the influence of baryons on galaxy formation which should be most important near the centers of galaxies. In the standard model slightly over-dense regions in a nearly uniform early DM distribution acted as seeds for future galaxies and clusters. Baryonic material subsequently fell into the potential wells established by the DM. The interplay of the baryons and DM through, e.g., adiabatic contraction and dynamical friction, has likely altered the spatial distributions of both quantities during galaxy formation and evolution $(27 ; 28 ;$;29; 30; 31; 32; 33). Hence, the DM profiles on galaxy scales can serve as important laboratories for studying galaxy formation and the nature of the DM particle.

Like all the different types of galaxies, giant elliptical galaxies have their own special properties that make them deserving of dedicated study. Unlike disk galaxies or the smallest dwarf elliptical galaxies, giant elliptical galaxies are sufficiently massive that multiple powerful techniques to measure their mass profiles are available for many systems. In particular, only for giant ellipticals can gravitational lensing and X-rays be used to complement the information available from stellar dynamics, the latter of which is also available for smaller galaxies. Also, in contrast to the smallest galaxies, every giant elliptical is thought to host a super-massive black hole $(\mathrm{SMBH})$ at its center, the formation of which is apparently intertwined with the host galaxy (34; 35). It is also becoming increasingly recognized that galaxies acquire their gas in two modes that are distinguished by whether or not the in-falling gas is heated (36; 37). Both modes can operate in the transition mass regime of giant elliptical galaxies $\left(10^{12}-10^{13} M_{\odot}\right)(38)$.

One of the advantages of using X-ray observations to probe DM and SMBHs in elliptical galaxies is that the hot X-ray-emitting interstellar medium (ISM) fills the three-dimensional galactic potential well, thus providing a continuous tracer from the galactic nucleus out to well past the optical half-light radius where stellar dynamical studies become very challenging. In order to translate an X-ray observation of the hot ISM into a gravitating mass profile and DM measurement, it is required that hydrostatic equilibrium holds to a good approximation throughout the region of interest; i.e., that the thermal gas pressure of the ISM balances the weight of the gas. With X-ray observations providing increasing evidence of morphologically disturbed elliptical galaxies, it is timely to review the accuracy of the approximation of 
hydrostatic equilibrium in X-ray determinations of their DM profiles. Understanding and quantifying deviations from hydrostatic equilibrium not only will lead to more accurate X-ray mass determinations, but any measured non-hydrostatic gas motions will provide clues to elliptical galaxy formation $(39 ; 36 ; 37 ; 38 ; 40 ;$; 41 ).

Since giant elliptical galaxies tend to be located in dense environments, it can be difficult to disentangle any DM associated with the elliptical galaxy from its parent halo. Naturally, most of the attention of X-ray studies has been devoted to those systems with the largest X-ray fluxes which has lead to an emphasis on elliptical galaxies located at the centers of the most massive galaxy groups and clusters. While all elliptical galaxies are of interest for DM studies, in this review we address galaxysized halos by focusing on the lower mass regime that is still accessible to detailed $\mathrm{X}$-ray study $\left(10^{12} \mathrm{M}_{\odot} \lesssim \mathrm{M}_{\mathrm{vir}} \lesssim 10^{13} \mathrm{M}_{\odot}\right)$ We restrict consideration to objects where a single giant elliptical galaxy dominates the stellar light, which at the upper end of the mass range under consideration are often classified as fossil groups (42; 43; 44) which are thought to be highly evolved, relaxed systems (45; 46; 47).

In the present review we focus on the constraints on DM in elliptical galaxies obtained using the current generation of X-ray observatories - Chandra, XMM, and Suzaku; constraints from the previous generation of X-ray satellites are reviewed by, e.g., (48; 49; 39). While we take care to mention relevant results obtained from other techniques, our review is not intended to be complete from the multi-wavelength perspective. The interested reader is urged to consult the reviews by (50; 51) and (52; 53) for discussions of the constraints on DM in elliptical galaxies obtained by stellar dynamics and gravitational lensing.

\section{Hydrostatic Models of the Hot ISM}

\subsection{Preliminaries}

The hot ISM of an elliptical galaxy $\left(T \sim 10^{7} \mathrm{~K} \sim 0.9 \mathrm{keV} / k_{B}\right)$ has properties that are extremely similar to the (hotter) intracluster medium (ICM) of a massive galaxy cluster (54; 55; 56). While the detailed radiation spectrum is described well by a collisionally ionized plasma of electrons and ions obeying the coronal approximation (57), the macro-structure of the hot ISM in an elliptical galaxy is for many purposes also described well by a simple monatomic ideal gas. We highlight four key features of the "hot gas" relevant for constructing hydrostatic models to measure the DM content in elliptical galaxies.

\section{Collisional Fluid}

In a fully ionized plasma, the equipartition timescale for electrons considering only their mutual Coulomb interactions is (58), 


$$
\begin{aligned}
\tau_{e-e} & =\frac{3 m_{e}^{1 / 2}\left(k_{B} T_{e}\right)^{3 / 2}}{4 \pi^{1 / 2} n_{e} e^{4} \ln \Lambda_{\mathrm{coul}}} \\
& =1.1 \times 10^{4}\left(\frac{T_{e}}{10^{7} \mathrm{~K}}\right)^{3 / 2}\left(\frac{n_{e}}{10^{-3} \mathrm{~cm}^{-3}}\right)^{-1} \mathrm{yr},
\end{aligned}
$$

where $m_{e}$ is the electron mass, $e$ is the electric charge, $k_{B}$ is Boltzmann's constant, $n_{e}$ is the electron number density, and $T_{e}$ is the electron temperature. For $T_{e}>4 \times 10^{5} \mathrm{~K}$ the Coulomb logarithm of an electron-proton plasma is (58),

$$
\ln \Lambda_{\text {coul }}=35.4+\ln \left[\left(\frac{T}{10^{7} \mathrm{~K}}\right)\left(\frac{n_{e}}{10^{-3} \mathrm{~cm}^{-3}}\right)^{-1 / 2}\right] .
$$

The mean free path of the electrons for Coulomb collisions is $\mathrm{v}_{e} \tau_{e-e}(59 ; 60 ;$; 61), where $\mathrm{v}_{e}=\sqrt{3 k_{B} T_{e} / m_{e}}$ is the rms thermal velocity of the electrons, so that,

$$
\begin{aligned}
\text { Coulomb mean free path } & =\frac{3^{1 / 2}\left(k_{B} T_{e}\right)^{2}}{4 \pi^{1 / 2} n_{e} e^{4} \ln \Lambda_{\text {coul }}} \\
& =243\left(\frac{T}{10^{7} \mathrm{~K}}\right)^{2}\left(\frac{n_{e}}{10^{-3} \mathrm{~cm}^{-3}}\right)^{-1} \mathrm{pc} .
\end{aligned}
$$

Because $m_{e}$ does not enter the above equation this expression also represents the mean free path for the protons (for $n_{e}=n_{p}$ and $T_{e}=T_{p}$ ). The electron and proton mean free paths are generally much smaller than the length scales of interest for DM studies indicating that the plasma may be treated as a collisional fluid. Still, in the outer regions near the virial radius, where $n_{e}$ is small, and therefore the Coulomb mean free path is large, it is possible the fluid approximation considering only Coulomb collisions may not always be justified. But the hot ISM of elliptical galaxies may typically possess tangled $\sim 1 \mu \mathrm{G}$ magnetic fields (62; 63). If so, the electron gyroradius, $r_{g}=\mathrm{v}_{\mathrm{e}} / \omega$, for angular gyrofrequency $\omega=e B / m_{e} c$,

$$
r_{g}=3.9 \times 10^{-11} Z^{-1}\left(\frac{T}{10^{7} \mathrm{~K}}\right)^{1 / 2}\left(\frac{m}{m_{e}}\right)^{1 / 2}\left(\frac{B}{1 \mu G}\right)^{-1} \mathrm{pc},
$$

is vastly smaller than the relevant length scales for DM studies. (The same is true for the proton gyroradius obtained by setting $m=m_{p}$.) Consequently, if the hot ISM contains (plausible) weak magnetic fields, then it is expected to behave as a collisional fluid on all length scales of interest for DM studies, allowing an isotropic pressure and temperature to be defined for any fluid element.

\section{Local Maxwellian Velocity Distribution}

In any fluid element in the hot ISM Coulomb collisions between electrons establish a Maxwellian velocity distribution with kinetic temperature $T_{e}$ on the time scale given by equation (1). This is much shorter than other time scales associated with the hot 
ISM in a relaxed elliptical galaxy. In general the ions can reach equilibrium with a kinetic temperature different from that of the electrons. But electron-ion collisions will establish equipartition on a time scale $\approx\left(m_{p} / m_{e}\right) \tau_{e-e} \approx 1870 \tau_{e-e} \approx 10^{7} \mathrm{yr}$ for the conditions typical of the hot ISM $(58)$. Only for galaxies currently undergoing strong dynamical disturbances, particularly in the central regions of some galaxies with AGN, might be evolving dynamically on a comparable time scale so that the electron and ion temperatures could be different. In relaxed elliptical galaxies, however, the hot ISM is expected to behave locally as a Maxwellian velocity distribution with a single kinetic temperature for the electrons and the ions. Therefore, the thermal pressure of the hot ISM is characterized by that of a monatomic ideal gas, $\rho_{\mathrm{gas}} k_{B} T / \mu m_{\mathrm{a}}$, where $\mu$ is the mean atomic weight of the gas and $m_{\mathrm{a}}$ is the atomic mass unit. For a fully ionized gas of solar abundances, $\mu=0.62$.

\section{Thermal Pressure Dominates Magnetic Pressure}

Observations of radio halos and relics in several massive galaxy clusters indicate that the ICM is magnetized with a field strength of $1-10 \mu \mathrm{G}$ (64). Unfortunately, elliptical galaxies are not observed to possess the large-scale diffuse radio emission observed in some massive clusters, and thus knowledge of their magnetic fields is derived from systems with embedded radio jets where the inferred $\sim \mu \mathrm{G}$ fields (62) may not be representative of the typical hot ISM. For fields of this magnitude, with $P_{\text {mag }}=B^{2} / 8 \pi$ and $P_{\text {therm }}=n_{\text {gas }} k_{B} T \approx 1.9 n_{e} k_{B} T$,

$$
\frac{P_{\text {mag }}}{P_{\text {therm }}}=0.015\left(\frac{B}{1 \mu G}\right)^{2}\left(\frac{T}{10^{7} \mathrm{~K}}\right)^{-1}\left(\frac{n_{e}}{10^{-3} \mathrm{~cm}^{-3}}\right)^{-1},
$$

which implies a minor, though very uncertain, contribution of magnetic fields to the pressure support of the hot ISM. Many theoretical models of inflowing gas in elliptical galaxies predict that small seed magnetic fields are amplified possibly to this level or larger (39, and references therein), although the small gas fractions observed at the centers of groups (65; 66) likely indicates that any hypothetical concentration magnetic fields, e.g., arising from the amplification owing to inflowing gas just described, has been dispersed by feedback. X-ray studies of DM in elliptical galaxies generally neglect the magnetic pressure, an assumption that can be tested indirectly by (1) comparing observed stellar mass-to-light ratios to theoretical stellar population synthesis models and (2) comparing mass profiles obtained by $\mathrm{X}$-ray methods with those obtained from stellar dynamics and gravitational lensing (88).

\section{Thermal Motions Dominate the Hot ISM in Relaxed Galaxies}

When the gravitational potential evolves on a time scale longer than the dynamical time, approximately given by the crossing time of the stars, 


$$
t_{\mathrm{cross}}=\frac{d}{\sigma}=3.9 \times 10^{7}\left(\frac{d}{10 \mathrm{kpc}}\right)\left(\frac{\sigma}{250 \mathrm{~km} \mathrm{~s}^{-1}}\right)^{-1} \mathrm{yr},
$$

where $d$ is the length scale of the region under consideration and $\sigma$ is the stellar velocity dispersion within that region, a time-independent description of the gravitational potential is appropriate. This quasi-static description of the gravitational potential is valid regardless of the dynamical state of the hot ISM, because the gas contributes negligibly to the total mass (gas+stars+DM) over most of the radial range within the virial radius of an elliptical galaxy. Radiative cooling of the hot gas acting alone cannot drive evolution on time scales smaller than the cooling time $\left(\approx 5 \times 10^{6} / n_{e}\right.$ yr for $T=10^{7} \mathrm{~K}$ and solar abundances, or $5 \times 10^{9} \mathrm{yr}$ for $n_{e}=10^{-3} \mathrm{~cm}^{-3}$ ) which is generally much longer than $t_{\text {cross }}$. But rapid evolution on a time scale $\lesssim t_{\text {cross }}$ is expected to prevail in the central regions of galaxies that have experienced recent, strong AGN feedback presumably generated in response to gas cooling. For our purposes, a relaxed elliptical galaxy is one in which the structure of the hot ISM is also evolving quasi-statically on a time scale longer than $t_{\text {cross }}$ described by a state of hydrostatic equilibrium, which applies provided any nonthermal gas motions (i.e., motions that are not associated with the random velocities that define the thermal gas pressure) are negligible compared to the sound speed,

$$
c_{s}=\left(\frac{\gamma P_{\mathrm{gas}}}{\rho_{\mathrm{gas}}}\right)^{1 / 2}=473\left(\frac{T}{10^{7} \mathrm{~K}}\right)^{1 / 2} \mathrm{~km} \mathrm{~s}^{-1}
$$

where $\gamma=5 / 3$ is the adiabatic index. This implies a sound crossing time similar to the stellar crossing time in equation (6). Turbulence is expected to be introduced into the hot ISM from merging and intermittent AGN feedback. Interesting limits on turbulent motions have been obtained via constraints on resonance scattering in the center ( $\lesssim 1 \mathrm{kpc}$ ) of the elliptical galaxy NGC $4636(67)$ and from Doppler broadening of the X-ray emission lines in a small number of elliptical galaxies (NGC 533, NGC 1399, NGC 4261, NGC 5044) from the sample of (68) suggesting that any turbulent motions present in the central regions of those systems are highly subsonic. While not expected to dominate, turbulent velocities may not be negligible since cosmological hydrodynamical simulations typically find that turbulence contributes up to $\sim 20 \%$ of the pressure support of the ICM in relaxed galaxy clusters $(69 ;$;0; 71; 72; 73). The irregular, subsonic $\mathrm{H} \alpha$ velocities observed at the centers of some elliptical galaxies also suggest turbulent velocities in the hot gas (74). It is unlikely that rotation dominates the ISM dynamics on large scales in elliptical galaxies given the lack of flattened disks observed (75), though modest rotational spin-up might occur in the innermost $(\lesssim 1 \mathrm{kpc})$ regions of some galaxies $(41)$. The expected inflow velocities arising from radiative cooling are negligible (39), and a recent study of galaxy-scale halos using a cosmological hydrodynamical simulation concludes that their hot ISM is generally quasi-hydrostatic $(76 ; 77)$. 


\subsection{The Equation of Hydrostatic Equilibrium: Usage Guidelines}

For the relaxed elliptical galaxies under consideration, hydrostatic equilibrium of a fluid element of hot ISM is expressed as the balance between forces per unit volume of gravity and gas pressure,

$$
\nabla P_{\text {gas }}=-\rho_{\text {gas }} \nabla \Phi
$$

where $\Phi$ is the gravitational potential, $P_{\text {gas }}$ is the pressure and $\rho_{\text {gas }}$ the density of the ISM. If non-thermal effects are significant that can be represented by a pressure $\left(P_{\mathrm{nt}}\right)$, such as for isotropic random turbulence or a magnetic field, then $P_{\mathrm{gas}}=P_{\mathrm{t}}+P_{\mathrm{nt}}$, where $P_{\mathrm{t}}$ is the thermal pressure. For the case of solid-body rotation, $\Phi$ can be replaced by an effective potential. For general rotation (and other ordered gas motions) the convective derivative term $(\mathbf{v} \cdot \nabla) \mathbf{v}$ in the Euler equation must be added to the left-hand side of equation (8). As remarked previously, the hydrostatic approximation is appropriate provided the non-thermal gas motions are subsonic.

In order to measure directly the magnitude of any non-thermal motions present in the hot ISM, it is necessary to measure precisely the Doppler shifts and broadening of emission lines which is beyond the capability of the current X-ray satellites - except possibly for the innermost regions of some galaxies noted above. Consequently, indirect methods are required to assess whether a galaxy is suitably relaxed for the hydrostatic approximation. Assessment of the morphology of the X-ray image is a convenient first step to locate candidate relaxed galaxies for the following reason. Because the total underlying stellar and DM distribution is expected to have a shape close to that of an ellipsoid, the gravitational potential is also expected to be approximately ellipsoidal, though rounder than the generating mass distribution. Since in hydrostatic equilibrium the X-ray emissivity traces the same three-dimensional shape as the potential for any temperature profile $(78 ; 79 ; 49$, also see 96 , galaxies that exhibit irregular, non-ellipsoidal features in their X-ray images might not be suitably relaxed for hydrostatic study. The existence of such asymmetrical features (e.g., substructure, cold fronts) does not guarantee large departures from hydrostatic equilibrium, as demonstrated by cosmological hydrodynamical simulations of clusters $(69 ; 70 ; 71 ; 72 ; 73)$, especially when the features are sufficiently localized so that they can be easily excluded from study $(80 ; 71)$.

Another important consideration in hydrostatic analysis is to insure that the X-ray emission that is attributed to hot ISM is not, in fact, heavily contaminated by unresolved discrete sources. A large number of the brightest discrete sources (mostly low-mass X-ray binaries - LMXBs) can be detected and excluded in nearby galaxies using the high spatial resolution of Chandra (see 81, and references therein); the lower resolution of XMM leads to far fewer detections, and only a few sources (if any) can be detected with Suzaku. Because the combined spectrum of unresolved LMXBs is spectrally harder than the hot ISM, the spectral signature of the hot ISM can be reliably extracted from the data provided it is not overwhelmed by the unresolved source component. The situation is more problematic in X-ray faint galaxies (having low ratios of X-ray-to-optical luminosity) like NGC 3379, where hydro- 
static mass estimates have been attempted $(82 ; 83)$ but the soft, diffuse X-ray emission attributed to hot ISM most likely originates almost entirely from cataclysmic variable stars and stellar coronae $(84 ; 85)$.

Once a suitably relaxed galaxy with sufficient emission from hot ISM has been identified, the accuracy of the hydrostatic equilibrium approximation can be assessed indirectly via consistency checks. First, one may examine if any wellmotivated hydrostatic model is able to provide an acceptable fit to the observed density and temperature profiles of the hot ISM. If an acceptable fit is obtained, the data are consistent with, though do not necessarily require, hydrostatic equilibrium. A poor fit - particularly one with sharp discontinuities in the density and temperature profiles - provides strong evidence for significant violations of the hydrostatic equilibrium approximation 1 . Second, the gravitating mass profile inferred from the $\mathrm{X}$-ray data can be compared to independent measurements using other techniques having different assumptions (stellar dynamics, gravitational lensing). When results obtained from different methods agree, it provides support for the underlying assumptions of each method and implies that systematic errors are well understood (see 98 ).

Hence, given the limitations of current X-ray data, a sensible approach to apply and assess hydrostatic equilibrium is the following:

\section{Guidelines for Applying Hydrostatic Equilibrium in Elliptical Galaxies:}

- Select systems which tend to have a regular, approximately cicular or elliptical $\mathrm{X}$-ray image morphology. If the image has asymmetric features, they should be preferably confined to spatial regions that are small compared to the region of interest; e.g., the inner region showing evidence of possible AGN feedback.

- Unresolved discrete sources should not dominate the X-ray emission. As a rough guide, at least half the X-ray luminosity within the optical half-light radius should originate from hot gas.

- Determine whether a hydrostatic model is able to provide an acceptable fit to the density and temperature profiles of the hot ISM over the region of interest.

- When possible, compare the mass profile obtained via the hydrostatic approximation with that obtained by an independent method; e.g., stellar dynamics, gravitational lensing. The hydrostatic equilibrium approximation is judged to be useful if it provides DM measurements of comparable (or better) quality than other techniques - or provides any information when no other technique is available.

The disturbed X-ray image of M 84 is a spectacular example (89) of the profound effect that AGN feedback can have on the hot ISM and is not a galaxy where hydro-

\footnotetext{
${ }^{1}$ Still, even in such cases the hydrostatic approximation might be useful. The core of M $87-\mathrm{a}$ system more massive than we consider in this review - possesses various spatial and spectral (86; 87) irregularities that are very likely associated with sizable non-hydrostatic gas motions. Yet (88) found that the gas is close to hydrostatic away from the regions of most significant disturbance.
} 

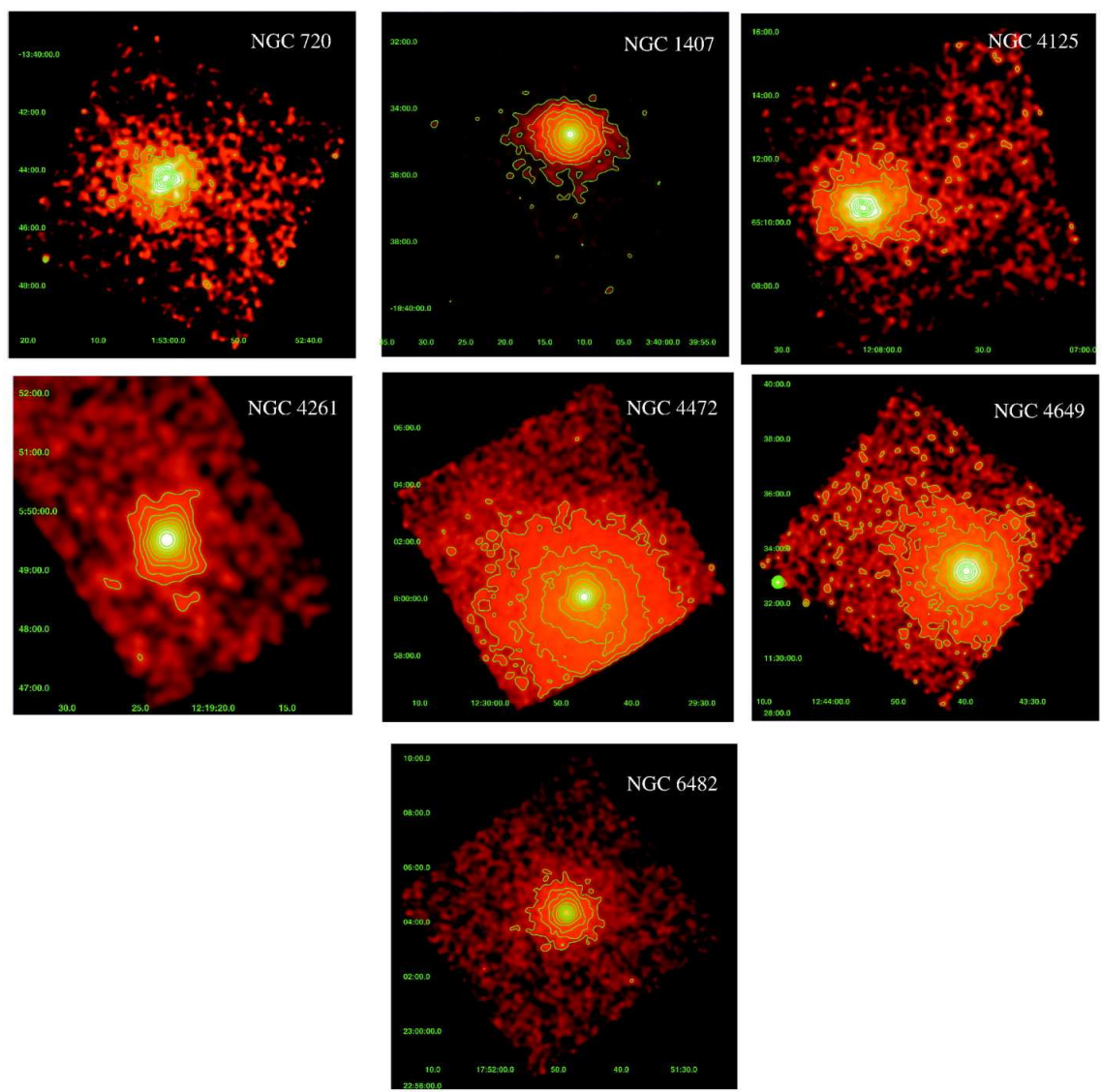

Fig. 1 A sample of seven nearby elliptical galaxies that are suitable for hydrostatic analysis, with each possessing regular morphologies as revealed by their Chandra images (94).

static equilibrium is expected to apply very accurately. But not all elliptical galaxies display such strong, large-scale irregularities in their X-ray images. In Figure 1 we display several elliptical galaxies with mostly regular images suitable for hydrostatic analysis. Two of the them, NGC 720 and NGC 4649, have very regular images deep into their cores (Figure 2). Another two, NGC 4472 and NGC 4261, possess low-level image fluctuations consistent with cavities associated with radio jets that do not greatly interfere with hydrostatic mass analysis. For several of these galaxies, radial entropy profiles have been computed $(90 ;$;1; 92) and found to increase monotonically with radius as also found in groups and clusters (93), indicating that the hot ISM is stable to convection and consistent with approximate hydrostatic equilibrium. 

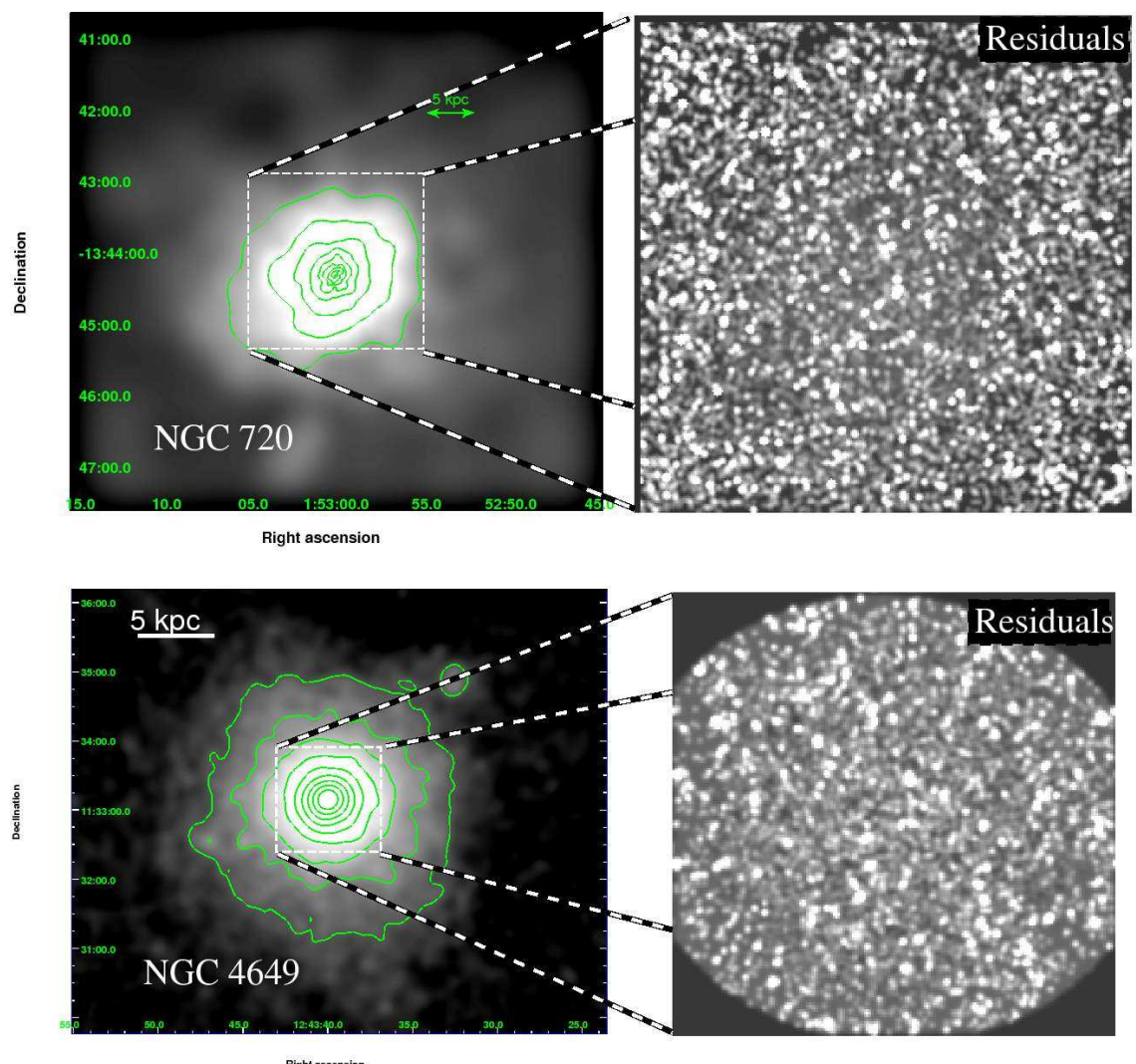

Fig. 2 Residual significance images of the central regions of NGC 720 (top, adapted from (92)) and NGC 4649 (bottom), indicating deviations from a smooth model fit to the X-ray isophotes 90 ; 92). Both galaxies appear to be relaxed and symmetrical at all scales accessible to the Chandra images.

\subsection{On the Incidence of Relaxed Elliptical Galaxies}

In a controversial study, Diehl \& Statler (95) investigated the X-ray and optical isophotal ellipticities in a heterogeneous sample of early-type galaxies to test whether they are consistent with hot ISM obeying hydrostatic equilibrium. The expected correlation between the ellipticities assumes that both hydrostatic equilibrium holds exactly and mass follows (optical) light, so that the stars define the shape of the gravitational potential and thus the shape of the X-ray emission. In an attempt to mitigate the impact of any DM halo, Diehl \& Statler restricted their analysis of each galaxy to within a small radius $-60 \%-90 \%$ of the optical half-light radius. When the expected correlation between the X-ray and optical isophotal ellipticities was not found, they concluded that hydrostatic equilibrium is not ubiquitous and, therefore, should never be assumed (even approximately) in mass analysis. They 
speculate that assuming hydrostatic equilibrium will always result in an error typically as large as the value of the mass measured; i.e., relaxed elliptical galaxies do not exist. While we agree (as detailed in the previous section) that care should be exercised in the application of hydrostatic equilibrium to the study of elliptical galaxies, we strongly disagree with their general conclusions about the accuracy of $\mathrm{X}$-ray mass measurements.

The observed lack of a correlation does not allow conclusions to be drawn with certainty for an individual object; it only indicates that hydrostatic equilibrium is unlikely to hold perfectly in a galaxy randomly drawn from their sample, provided it is chosen without any consideration of its morphology. As noted above, hydrostatic equilibrium as expressed by equation (8) is an approximation and is not expected to hold exactly given especially the impact of turbulence on the hot ISM induced by past mergers and AGN feedback. In other words, no elliptical galaxy is expected to be perfectly relaxed. The objects displayed in Figures 1 and 2 are among the most relaxed elliptical galaxies possessing high-quality X-ray data. But Diehl \& Statler's sample also contained galaxies with pronounced large-scale asymmetries that we would not recommend the routine application of hydrostatic mass methods following the guidelines discussed previously (e.g., NGC 4636: (96) and M 84: (89)), and since they focused on the smallest scales (where X-ray point-source removal is most challenging and where AGN-driven disturbances are most serious), in contrast to the larger scales that are most important for DM analysis, the implications of their study for DM measurements of morphologically relaxed systems are dubious and provide no basis for claiming typical mass errors $\gtrsim 100 \%$ on all scales in such systems.

An example of how conclusions regarding the center of a galaxy need not apply on larger scales is provided by NGC 5044. In this system Diehl \& Statler measured an X-ray ellipticity $(0.41 \pm 0.08)$ greatly exceeding the optical value $(0.07 \pm 0.01)$, which cannot be reconciled easily with hydrostatic equilibrium (unless the gas is also rapidly rotating). But the X-ray ellipticity measurement is confined to the region within the optical half-light radius $(<4 \mathrm{kpc})$ containing obvious disturbances presumed to be associated with AGN feedback (97; 98; 99). (We note, however, that there is recent evidence that turbulent motions of the hot ISM are negligible compared to the sound speed in the center of NGC 5044 (68).) The X-ray emission can be traced out at least to several hundred $\mathrm{kpc}$, about one-third of the virial radius (100; 101). Outside of the very central region discussed by Diehl \& Statler, the $\mathrm{X}$-ray isophotes are largely symmetric and nearly circular, and acceptable fits with hydrostatic models are obtained (65).

Now turning our attention to the central regions within the optical half-light radius relevant to the study of SMBHs ( 97 , we remark that additional factors can introduce scatter into the correlation between the isophotal ellipticities generated by the stars and hot ISM. As noted above, Diehl \& Statler assume that the mass traces the same shape as the optical light, whereas both X-ray and optical observations suggest DM distributed differently from the stars typically contributes 20\%-30\% within the half-light radius in giant elliptical galaxies (see 44. Furthermore, their novel technique to create X-ray images of diffuse hot ISM strictly requires there are no spatial variations in the spectral properties; 
i.e., every galaxy is assumed to be isothermal with constant metallicity throughout, in conflict with observations of many galaxies possessing high-quality X-ray data $(102 ; 103 ; 104 ; 105 ; 82 ; 106 ; 107 ; 108)$. Finally, ISM rotation might be important in some galaxies and yet have little relation to stellar rotation because slowly rotating inflowing gas at large radius might be spun up to dynamically relevant velocities if angular momentum is conserved. But rotational spin-up is not guaranteed for every galaxy, and instead the gas can be outflowing gently in a subsonic wind greatly reducing the impact of any rotation initially provided by the stars - see discussion in (39).

Before leaving this topic, we caution that a statistical appraisal of the properties of the hot ISM in any sample of elliptical galaxies should consider the sample selection criteria. Our impression of the X-ray properties of elliptical galaxies is obtained within the context of what are essentially optically selected samples. But for the study of ISM properties, it is important to select the sample according to the ISM luminosity and not the total $\mathrm{X}$-ray luminosity which also contains the emission from discrete sources associated with the stellar population. Diehl \& Statler follow the common approach of studying a "heterogeneous" sample of elliptical galaxies, which essentially amounts to collecting all objects in the archives with data of sufficient quality. This procedure resulted in a sample containing some well known objects with extended, X-ray luminous ISM (e.g., NGC 4472) and some where virtually all the X-ray emission can be attributed to discrete sources (e.g., NGC 3379 $-(84 ;$ (85)).

It is instructive, therefore, to consider a sub-sample extracted from their sample of 54 galaxies that is selected based on the X-ray luminosity of the diffuse gas. Using the tabulated diffuse gas luminosities published by Diehl \& Statler, we construct such a sub-sample by excluding massive group/cluster systems (gas $L_{\mathrm{x}}>3 \times$

$10^{42} \mathrm{erg} \mathrm{s}^{-1}$ ) and gas-poor galaxies ( $2 \sigma$ lower limit on gas $L_{\mathrm{x}}<1 \times 10^{40} \mathrm{erg} \mathrm{s}^{-1}$ ) resulting in a sample of 26 galaxies, give or take a few depending on how we interpret the quoted lower or upper limits for some systems. This sub-sample includes the seven galaxies shown in Figure 1 , as well as several other systems with generally regular X-ray images and where hydrostatic equilibrium previously has been profitably employed - NGC 533, NGC 1399, NGC 1404, NGC 3923, NGC 4555, NGC 5044. The sample also contains well-known disturbed systems such as M 84, NGC 4636, and NGC 5846, which are expected to deviate from hydrostatic equilibrium. In sum, an ISM-selected sub-sample culled from Diehl \& Statler's heterogeneous sample indicates that approximately $50 \%$ of the elliptical galaxies are sufficiently relaxed for hydrostatic analysis.

\subsection{Methods}

The variety of techniques that have been developed to apply hydrostatic equilibrium to the study of the mass distributions in elliptical galaxies (as well as galaxy clusters) can be divided into two broad classes that will be denoted here as smoothed 
inversion and forward-fitting. Whereas the forward-fitting approach assumes parameterized models for the mass profile and one thermodynamical variable of the gas (e.g., temperature profile), smoothed inversion instead introduces one or more arbitrary parameters to guarantee a smooth (physical) mass profile. The decision of whether or not to adopt a particular approach depends on several factors which we discuss below. Generally, the smoothed inversion approach is preferred if one is primarily interested in measuring the value of the enclosed mass within some radius without making any reference to an assumed (parameterized) mass model. If instead one desires to test the viability of an assumed mass model (e.g., power-law, $\mathrm{NFW}$ ) and to measure its parameters for comparison to other galaxies and theory, the forward-fitting approach is preferred. Whenever possible both approaches should be employed to assess the magnitude of any biases.

We note that in the following it is generally assumed that the gas density and temperature profiles (projected or deprojected) of the hot gas have been already obtained via an analysis of the spatially resolved X-ray spectra. The hydrostatic models then use these measured profiles to constrain the mass distribution. This decoupling of the spectral fitting and mass modeling, though convenient and generally less computationally expensive, is not necessary. The formalism of both of these approaches is reviewed in Appendix B of (65).

In the following subsections, we outline in more detail how the two broad model classes are implemented.

\subsubsection{Smoothed Inversion}

\section{Pros:}

- Mass measured without reference to an input parameterized model.

- Does not require an input parameterized model for the gas temperature, density, pressure, or entropy.

\section{Cons:}

- Deprojection requires a high degree of symmetry (usually spherical).

- Cannot self-consistently account for the projection of galaxy emission from any radii outside of the observed data range or, generally, extrapolate the hydrostatic model outside the data range.

- Arbitrary smoothing required to obtain a physical (monotonically increasing) mass profile. The amount of smoothing increases for lower data quality.

The aim of the smoothed inversion approach is to measure the mass profile without reference to an assumed (parameterized) model for the mass distribution or any quantity associated with the hot ISM (gas temperature, density, pressure, or entropy). For this to be achieved, the three-dimensional distribution of the hot ISM 
must be obtained by non-parametrically deprojecting the data on the sky. This is only possible if the ISM has a high degree of symmetry, and therefore spherically symmetric deprojection using the well-known "onion peel" method originally developed for clusters is almost always adopted $(109 ; 110)$. Since the onion peeling starts from the outer edge of the galaxy and works its way inward, such deprojection formally requires that the data span the entire size of the galaxy. As this is not generally the case, ad hoc prescriptions are usually employed to estimate the projection of gas from radii larger than the extent of the available data $(111 ; 112)$.

Because no global mass model is assumed, the value of the mass determined within a given shell can be negative due to noise in the data (amplified by deprojection). To circumvent this problem so as to achieve a mass profile that increases monotonically with radius, the inversion procedure must smooth the gas quantities (e.g., temperature and density) and the mass profile itself. The amount and type of smoothing is arbitrary, and typically as the data quality decreases the amount of smoothing required to achieve interesting constraints generally increases. As discussed below, types of smoothing include (1) the use of ad hoc smooth parameterized models for the gas density and temperature, (2) assuming the ISM is constant in spherical shells so that the widths of the shells set the smoothing scales, and (3) one or more parameters in a Bayesian prior. While the smoothing can be well-motivated, it necessarily introduces additional assumptions, usually expressed by additional parameters that must be determined empirically by the data. Consequently, we believe that the terms "non-parametric" or "model-independent" sometimes used to describe smoothed inversion methods are misleading and should be avoided.

In the context of DM studies, smoothed inversion is best suited to providing a measurement of the enclosed mass within a particular radius without assuming a specific parameterized mass model; e.g., measuring $M_{500}$ for the purpose of establishing the mass function of elliptical galaxies. But if one also desires to fit and measure the parameters of a mass model (e.g., global power-law slope, NFW concentration parameter), the ad hoc smoothing adds a barrier between the data and model which is not present in the forward-fitting approach that is more naturally suited for this problem.

\section{Traditional Approach: Parameterized Density and Temperature Profiles}

For a spherically symmetric galaxy where the hot ISM is supported by ideal gas pressure, equation (8) can be solved for the total gravitating mass (stars, gas, and $\mathrm{DM})$ as a function of the gas density $\left(\rho_{\mathrm{gas}}\right)$ and temperature $(T)$,

$$
M(<r)=-\left[\frac{r k_{\mathrm{B}} T}{\mu m_{\mathrm{a}} G}\right]\left[\frac{d \ln \rho_{\mathrm{gas}}}{d \ln r}+\frac{d \ln T}{d \ln r}\right],
$$

where $k_{\mathrm{B}}$ is Boltzmann's constant, $\mu$ is the mean atomic weight of the gas, $m_{\mathrm{a}}$ is the atomic mass unit, and $G$ is Newton's gravitation constant. Beginning with M 87 over thirty years ago $(113 ;$;14), this equation has been widely applied to galaxies and 
clusters traditionally by inserting smooth parameterized functions for the density and temperature into equation (9). The ad hoc parameterized functions do not guarantee a physical mass profile that increases monotonically with increasing radius, and their use offsets any gain in rigor from not assuming a specific mass model. Thus, while this traditional approach has the advantage of being conceptually simple and computationally inexpensive, it is best employed as a rough estimate and a check on other methods.

\section{Smoothing by Binning}

The derivatives in equation (96) may instead be computed directly from the density and temperature values measured in adjacent radial bins rather than by assuming any parameterized models. Because of bin-to-bin statistical fluctuations in the density and temperature (or pressure) profiles, increased binning (i.e., smoothing) of the radial profile is generally required to produce an approximate monotonically increasing mass profile. This method has only been attempted for the brightest galaxy clusters (115; 116), since only for data of the highest quality can interesting results be obtained without resorting to heavy binning of the data.

Recently, Nulsen et al. (117) have proposed a scheme that obviates the need for explicit computation of the derivatives by assuming that within any spherical shell the gravitating matter density and gas temperature are constant. By requiring that the gas pressure be continuous across shell boundaries, the gas density is then computed within any shell using an isothermal solution of equation (8). But the pressure continuity condition involves the difference in the gravitational potential between adjacent shells, which is related to a derivative (and is the argument of an exponential). Consequently, the mass profile derived from this procedure remains subject to similar bin-to-bin statistical fluctuations that occur when the derivatives in equation (9) are computed explicitly. To improve the smoothness of the derived mass profile, Nulsen et al. advocate requiring that the gravitating matter density decrease monotonically with increasing radius.

\section{Bayesian Inversion with Smoothing Prior}

A very general approach to smoothed inversion has been proposed by Das et al. (118), which is based on the seminal works of Merritt \& Tremblay (119) and Magorrian (120). Das et al. enforce smoothness via a Bayesian prior which penalizes large second derivatives between radial bins in the temperature, circular velocity (hence the mass), and pressure gradient. The magnitude of the smoothing is controlled by

a parameter $\lambda$, the value of which is arbitrary and depends on both the number of radial bins and the model grid spacing. For convenience Das et al. use simulations to select a fixed $\lambda$ for all galaxies. Since the sensitivity of the derived mass profile to $\lambda$ should be investigated for any galaxy, a reasonable extension would be to define a prior for $\lambda$ and then marginalize over $\lambda$ for each system. 


\subsubsection{Forward-Fitting}

\section{Pros:}

- No formal restrictions on galaxy geometry (though spherical symmetry usually assumed).

- Allows for self-consistent extrapolation of hydrostatic model and projection of galaxy emission to and from any radii outside the data range.

\section{Cons:}

- Requires an input parameterized model for the mass.

- Requires an input parameterized model for one of the following thermodynamic quantities: gas temperature, density, pressure, or entropy.

The forward-fitting approach uses parameterized models for the mass and one thermodynamic variable of the hot ISM (density, temperature, pressure, or entropy). Because the galaxy is completely specified by the models, in principle any geometrical configuration can be accommodated, though spherical symmetry is usually assumed. The model can be extrapolated to radii outside the available data range allowing for self-consistent treatment of the projection of the ISM of the entire galaxy. Forward-fitting can be applied to data that have been deprojected (as with smoothed inversion) or directly to the data on the sky. The ability to fit projected models to data on the sky is especially important for lower quality, noisy data whose integrity would be unacceptably degraded by deprojection noise.

This approach is preferred when it is desired to test the viability of a particular mass model and to measure its parameters. If the assumed model is incorrect, then the measured mass at a given radius may differ systematically from the true value. It is therefore necessary to explore multiple mass models to gauge the magnitude of any bias. Since the measured mass may be sensitive to the assumed mass model, when possible results using forward-fitting should be compared to those from smoothed inversion.

Prior to Chandra and XMM, when accurate spatially resolved temperature profiles were only available for a few of the most massive galaxies, it was customary to make strong assumptions about the temperature profile in order to obtain an interesting constraint on the DM. Typically the gas was assumed to be isothermal or that it obeyed a polytropic equation of state $\left(P_{\mathrm{gas}} \propto \rho_{\mathrm{gas}}^{\gamma}\right)$. The most widely used of these approaches is the well-known isothermal "beta model" (121; 122; 123). While such procedures still can be useful for obtaining DM estimates from observations of low data quality, we focus our discussion on methods intended to exploit accurate spatially resolved temperature profiles, as such data are now more widely available. 


\section{Density-Based}

If an assumption is made about the form of the gas density profile, e.g., that it is described by a parameterized function, then taken together with an assumed parameterized mass model the equation of hydrostatic equilibrium (equation 8) may be solved to give the temperature,

$$
T(r)=T_{0} \frac{\rho_{\mathrm{gas}, 0}}{\rho_{\mathrm{gas}}(r)}-\frac{\mu m_{\mathrm{a}} G}{k_{\mathrm{B}}} \frac{1}{\rho_{\mathrm{gas}}(r)} \int_{r_{0}}^{r} \rho_{\mathrm{gas}}(r) \frac{M(<r)}{r^{2}} d r,
$$

where $T_{0}=T\left(r_{0}\right)$ and $\rho_{\text {gas }, 0}=\rho_{\text {gas }}\left(r_{0}\right)$ evaluated at some reference radius $r_{0}$. Here and below we assume spherical symmetry unless noted otherwise (i.e., in 6 . The parameters of the mass and density models and the normalization $T_{0}$ are then constrained by the observation. As with all the types of forward-fitting approaches we discuss, it is common practice first to measure the temperature and density profiles from fitting the spectra and then constrain the free parameters of the hydrostatic model via a simultaneous fit to the measured profiles. Although the gas contribution to the total mass $M(<r)$ in elliptical galaxies can generally be neglected until one approaches close to the virial radius, a self-consistent hydrostatic model should include it. For these density-based models this is straightforward since the gas density profile is assumed, and therefore the gas mass profile is defined before the temperature profile is computed.

An important variation of the density-based approach was proposed by Fabian and colleagues $(109 ; 110 ; 124 ; 125)$ which exploits the relatively high statistical quality of the X-ray surface brightness profile. Sometimes referred to as the "Cambridge Method", this approach assumes spherical symmetry and analytically deprojects the surface brightness using the onion peel procedure to give the threedimensional emissivity profile, $\varepsilon \propto \rho_{\text {gas }}^{2} \Lambda(T, Z)$, where $\Lambda(T, Z)$ is the plasma emissivity which depends on temperature $T$ and metal abundances $Z$ and is integrated over the relevant (broad) wave band. If $\Lambda(T, Z)$ is assumed to be constant over the galaxy, then $\rho_{\text {gas }}(r) \propto \sqrt{\varepsilon(r)}$. Because of the relatively high statistical quality of the surface brightness (and emissivity), the density profile derived in this way usually can be evaluated in much finer spatial bins than is possible for the temperature profile determined from detailed spectral fitting. This allows the finely binned density profile to be treated as a continuous function by interpolating values between the bins, which can then be inserted (along with a parameterized mass model) into equation (10) to predict the temperature profile for comparison to the values measured from the spectra.

It is a significant advantage of the Cambridge Method that it does not require for input an assumed, possibly oversimplified, parameterized model for the density profile. Still, the density profile generated assuming a constant $\Lambda$ leads to hydrostatic solutions that are not self-consistent in the presence of temperature and metallicity gradients. This effect is not expected to be large because variations in $\Lambda$ within a galaxy or cluster are probably no more than $\sim 20 \%$ which translate to inferred density differences of only $\sim 10 \%$. In principle, the best-fitting temperature pro- 
file could be used to define a new spatially varying $\Lambda$ from which a new density profile could be computed. The process could be iterated to achieve improved selfconsistency.

\section{Temperature-Based}

If an assumption is made about the form of the temperature profile, then in conjunction with a parameterized mass profile the equation of hydrostatic equilibrium (equation 8) may be solved for the gas density,

$$
\rho_{\mathrm{gas}}(r)=\rho_{\mathrm{gas}, 0} \frac{T_{0}}{T(r)} \exp \left[-\frac{\mu m_{\mathrm{a}} G}{k_{\mathrm{B}}} \int_{r_{0}}^{r} \frac{1}{T(r)} \frac{M(<r)}{r^{2}} d r\right],
$$

where spherical symmetry is again assumed. This approach can be especially useful when the temperature profile is poorly constrained by the data. Simple profiles (constant, power-law) can be adopted in order to explore their impact on the inferred mass profile. Unlike the density-based case, the gas mass contribution to $M(<r)$ cannot be included fully self-consistently in the fit. However, because the gas mass can be treated as a small perturbation, an iterative procedure can be employed; i.e., after first taking the gas mass contribution to $M(<r)$ to be zero, use the resultant best-fitting $\rho_{\text {gas }}(r)$ to compute a fixed gas mass profile that is added to $M(<r)$ and then fit again.

\section{Entropy-Based}

Recent studies have suggested that the entropy is the logical thermodynamic variable for which to assume a parameterized model (90; 91; 126; 127). Consistent with the hydrostatic equilibrium approximation is the requirement that the hot gas be stable against convection at every radius. Convective stability provides an important additional constraint on the mass profile that has been used in the past to set a robust lower limit on the total masses of galaxy clusters (128). For an ideal gas equation of state the first law of thermodynamics can be solved to yield the specific entropy, $s=\left(3 k_{\mathrm{B}} / 2 \mu m_{\mathrm{a}}\right) \ln \left(T \rho_{\mathrm{gas}}^{-2 / 3}\right)+$ constant. It is conventional for studies of the hot gas in galaxies and clusters to remove the logarithm and the numerical factor to define the quantity, $S \equiv\left(k_{\mathrm{B}} / \mu m_{\mathrm{a}}\right) T \rho_{\mathrm{gas}}^{-2 / 3}$, which we will refer to as the "entropy" in our discussion.

If parameterized models are assumed for both the entropy and mass profiles, then the equation of hydrostatic equilibrium can be rewritten so that,

$$
\frac{d \xi}{d r}=-\frac{2}{5} \frac{G M(<r)}{r^{2}} S^{-3 / 5}, \quad \xi \equiv P^{2 / 5} .
$$


Solving this equation for $\xi$ gives the profiles of gas density, $\rho_{\text {gas }}=(P / S)^{3 / 5}=$ $S^{-3 / 5} \xi^{3 / 2}$, and temperature, $k_{\mathrm{B}} T / \mu m_{\mathrm{a}}=S^{3 / 5} P^{2 / 5}=S^{3 / 5} \xi$, which are compared to the observation to constrain the parameters of the input $S$ and $M(<r)$ models. Since, however, the total mass $M(<r)$ also includes the gas mass which depends on $\xi$, equation (12) can be solved for $\xi$ by direct integration only if the gas mass is negligible with respect to the mass of the stars and DM. To include the gas mass self-consistently, equation (12) can be differentiated and rearranged to yield,

$$
\frac{d}{d r}\left(r^{2} S^{3 / 5} \frac{d \xi}{d r}\right)+\frac{8 \pi r^{2} G}{5} S^{-3 / 5} \xi^{3 / 2}=-\frac{8 \pi r^{2} G}{5}\left(\rho_{\text {stars }}+\rho_{\mathrm{DM}}\right)
$$

where use has been made of the relation $d M(<r) / d r=4 \pi r^{2}\left(\rho_{\text {stars }}+\rho_{\mathrm{DM}}+\rho_{\mathrm{gas}}\right)$. The two boundary conditions for this second-order differential equation require specifying the values of $\xi$ and $d \xi / d r$ at some radius. The value of $\xi$ amounts to specifying the pressure at some radius, which is just a normalization constant determined by the fitting of the density and temperature profiles. As discussed by (90), because the gas mass makes a negligible contribution to the total mass at small radius, equation (12) can be evaluated at such a small radius to give the $d \xi / d r$ boundary condition.

The Schwarzschild criterion for convective stability requires that the entropy increases monotonically with increasing radius, consistent with observations of relaxed elliptical galaxies, for which the entropy profiles are well described by simple models of a constant with a broken power-law $(90 ;$; 91 ; 92). The Schwarzschild criterion also effectively limits the magnitude of any temperature gradient. Stronger restrictions on the temperature gradient potentially could be enforced by MTI/HBI instabilities (129; 130) that develop in the weakly magnetized plasma characteristic of the hot gas in a galaxy cluster. These instabilities arise when heat and momentum transport occurs only along magnetic field lines, which may happen in regions of a plasma where the gyroradius is much smaller than the the Coulomb mean free path (such as for an elliptical galaxy - see 2.1 . But since these instabilities are expected to be weaker for the lower temperatures of elliptical galaxies, and they are also suppressed by small amounts of turbulence (131; 132), their relevance to elliptical galaxies is unclear.

Whereas gas density and temperature profiles display a large range of behavior, a robust result of cosmological simulations considering only gravity is that the radial entropy profile follows a power-law, $S(r) \sim r^{1.1}$ (133; 134$)$. Since the cooling and feedback processes that would modify this relation are expected to be most significant closer to the centers of halos, the $S(r) \sim r^{1.1}$ relation provides a physically well-motivated asymptotic relation that is particularly useful when extrapolating the hydrostatic model outside the data range (e.g., computing model projections). 

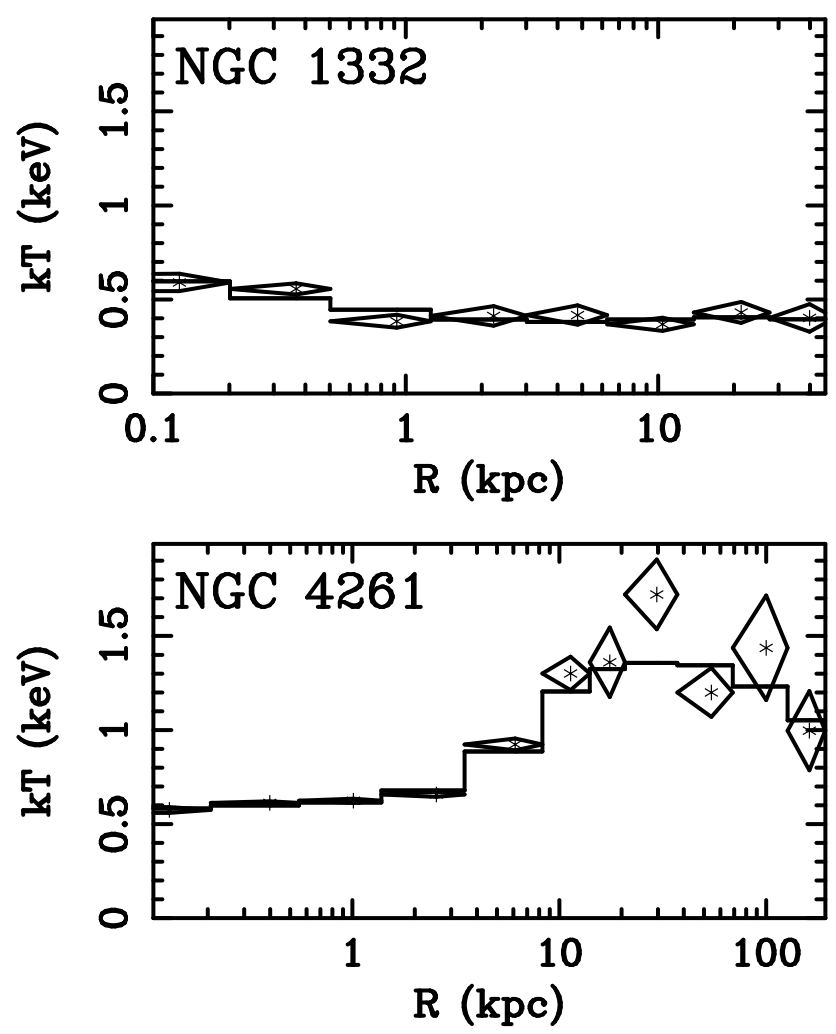

Fig. 3 Chandra temperature profiles of two galaxies from (91). The top panel shows the centrally rising profile of NGC 1332 characteristic of galaxies with $M_{\mathrm{vir}} \lesssim 10^{13} M_{\odot}$ and average temperature $k_{\mathrm{B}} T \sim 0.5 \mathrm{keV}$. The bottom panel shows the centrally falling profile of NGC 4261 characteristic of galaxies with $M_{\text {vir }} \gtrsim 10^{13} M_{\odot}$ and average temperature $k_{\mathrm{B}} T \sim 1 \mathrm{keV}$. The $K$-band half-light radii are 2.7 and $3.4 \mathrm{kpc}$, respectively, for NGC 1332 and NGC 4261.

\section{Is DM Required?}

Prior to the launches of Chandra and XMM, the principal limiting factor for most $\mathrm{X}$-ray studies of DM in elliptical galaxies was the temperature profile. (Nonspherical DM constraints, which are largely insensitive to the temperature profile, can be more affected by unresolved discrete sources $(138 ; 139 ; 140)$ - see $\$ 6$. In most cases isothermality was assumed, but the possibility of large radial temperature gradients meant that no firm conclusions on DM could be obtained for most galaxies (48). For a small number of the brightest, hottest $\left(k_{\mathrm{B}} T \sim 1 \mathrm{keV}\right)$ most massive (few $\times 10^{13} \mathrm{M}_{\odot}$ ) galaxies, ROSAT was able to place interesting constraints on the temperature profiles, from which large amounts of DM were inferred: NGC 1339 (141), NGC 4472 (142; 143), NGC 4649 (143), and NGC 5044 (100). 

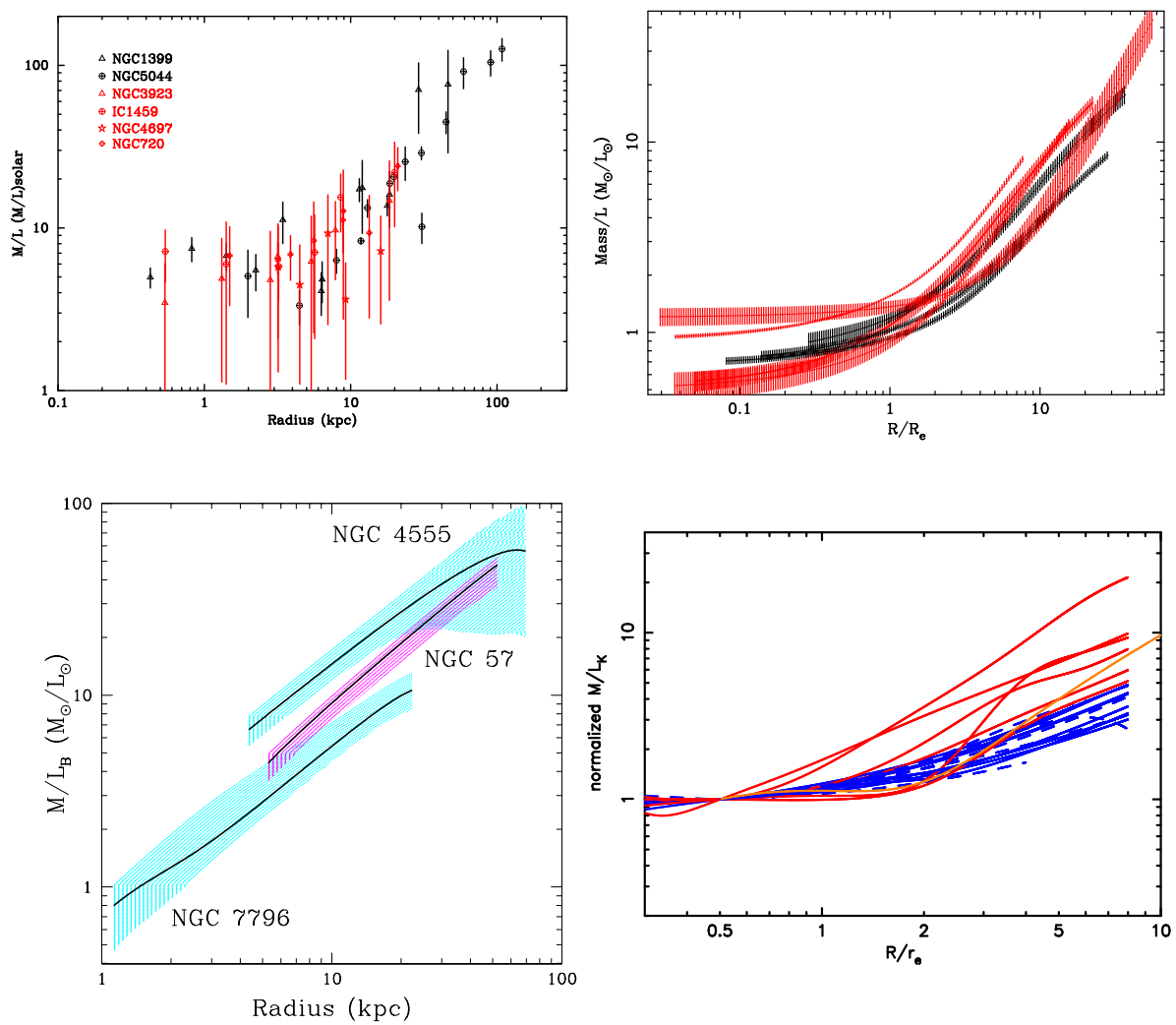

Fig. 4 Representative total mass-to-light $(M / L)$ ratios of elliptical galaxies obtained from Chandra and $X M M$ observations clearly showing rising $M / L$, and thus the need for DM, outside of $1-2$ optical half-light radii. (Top Left) Clear confirmation of rising $M / L$ in the massive galaxies NGC 1399 and NGC 5044 (82) found previously with ROSAT observations. (Top Right) Results for the galaxies displayed in Figure 1 obtained by (94). The black regions correspond to the galaxies NGC 720, NGC 4325, and NGC 6482 that have $M_{\mathrm{vir}} \lesssim 10^{13} M_{\odot}$ while the red regions correspond to the galaxies NGC 1407, NGC 4472, NGC 4649, and NGC 4261 that have $M_{\mathrm{vir}} \gtrsim 10^{13} M_{\odot}$. Each result shown reflects a $1 \sigma$ confidence region. (Bottom Left) $1 \sigma$ error regions for three galaxies from (135; 136), where isothermal temperature profiles have been adopted for NGC 57 and NGC 7796. (Bottom Right) Results from (137) for $M / L$ profiles of galaxies with temperature profiles that fall toward the center (red lines) and for others (blue lines).

Now accurate temperature profiles have been measured with Chandra and XMM for many galaxies (Figure 3). The shapes of the radial temperature profiles of earlytype galaxies can be separated into roughly two classes $(82 ;$;94; 137): (1) those with profiles that rise toward the center, corresponding to galaxies with $M_{\text {vir }} \lesssim 10^{13} M_{\odot}$ and average temperature $k_{\mathrm{B}} T \sim 0.5 \mathrm{keV}$, and (2) those with profiles that fall toward 


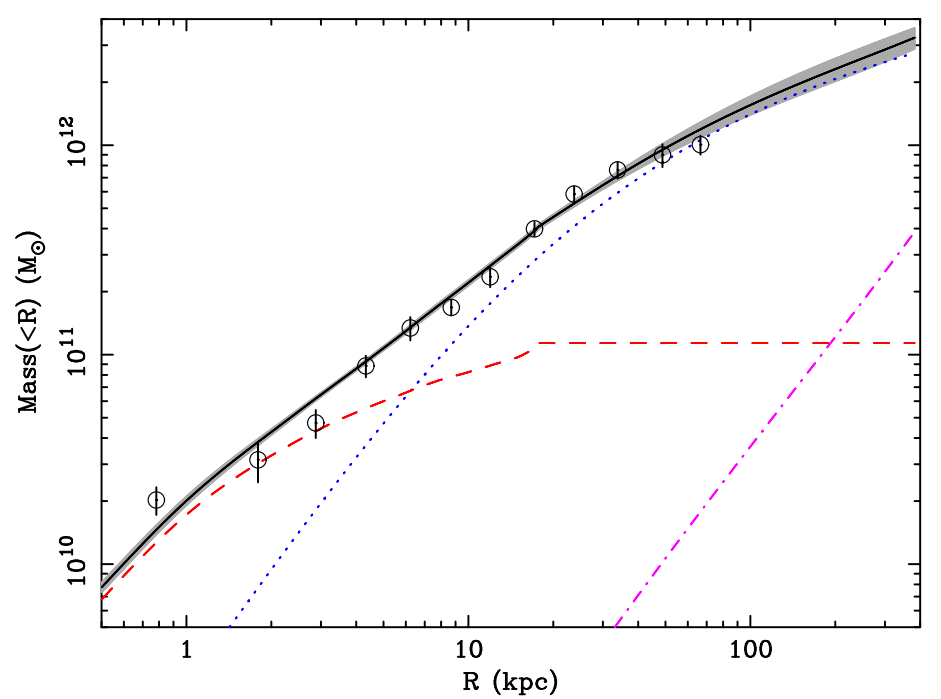

Fig. 5 Radial mass profile of NGC 720 obtained by (92) using deep observations with Chandra and Suzaku. The solid (black) line indicates the total enclosed mass, the dashed (red) line indicates the stellar mass, the dotted (blue) line is the DM contribution, and the dash-dot (magenta) line is the gas mass contribution. The grey shaded regions indicate the $1 \sigma$ error on the total mass distribution obtained using the entropy-based forward-fitting method. Overlaid are a set of data points derived from an independent analysis using the traditional smoothed inversion approach; i.e., the model indicated by the shaded region was not obtained by fitting these data points. Notice that DM is clearly required for radii larger than $\sim 5 \mathrm{kpc}$, about $2 R_{e}$, where $R_{e}=3.1 \mathrm{kpc}$ is the $K$-band halflight radius.

the center, corresponding to galaxies with $M_{\mathrm{vir}} \gtrsim 10^{13} M_{\odot}$ and average temperature $k_{\mathrm{B}} T \sim 1 \mathrm{keV}$. These temperature profiles enable accurate DM constraints for many galaxies, including lower mass systems (few $\times 10^{12} \mathrm{M}_{\odot}$ ).

Equipped with accurate temperature profiles from Chandra and $X M M$, precise constraints on mass-to-light ratios $(M / L)$ have now been obtained for many galaxies $(144 ; 135 ; 82 ; 94 ; 65 ; ; 136 ; 145 ; 146 ;$ 90; 91; 137; 92); here $M$ is the total mass and $L$ is the stellar luminosity, each computed within the same radius. Generally, for those galaxies with good constraints on the temperature profile out to a large radius, the data exclude a constant $M / L$ profile at high significance, instead requiring that $M / L$ rises with increasing radius outside of 1-2 optical half-light radii (Figure 4). The failure of the constant $M / L$ model provides strong evidence for DM.

It should be stressed that the current generation of X-ray satellites is able for the first time to provide excellent constraints on DM in lower mass galaxies with average temperature $k_{\mathrm{B}} T \sim 0.5 \mathrm{keV}$. Combining data from deep observations with Chandra and Suzaku, (92) obtained strong constraints on the mass profile of NGC 720 (see Figure 5) which was found to have a total mass $\left(M_{2500}=1.6 \pm 0.2 \times 10^{12} M_{\odot}\right)$ similar to that of the Milky Way. 
This X-ray evidence for DM determined individually for a relatively small number of elliptical galaxies ( $\sim 15$, depending on the upper mass limit) is complemented by evidence from large statistical studies in the optical. Weak lensing $(147 ; 148 ; 149 ; 150)$ and the dynamics of satellite galaxies (151) consider the accumulated data from a very large number of galaxies and find that the average halo mass $\sim 10^{12} M_{\odot}$ clearly exceeds that which can be attributed to the stars. As for constraints on individual systems, strong evidence for DM using sophisticated, orbit-based stellar dynamical models has been found recently for NGC 4649 (152), although the mass somewhat exceeds that inferred by X-rays, a point to which we shall return in more detail in $₫ 8$.

\section{Radial DM Profile}

Although the need for DM is now established for many elliptical galaxies, the X-ray data do not as of yet clearly favor a specific model for the radial profile. In particular, both the NFW and $\sim r^{-2}$ DM density profiles are consistent with available data. The NFW profile provides a useful means to compare observed DM halos to those predicted by cosmological models. The two parameters which specify the NFW profile are the concentration $c_{\Delta}$ and halo mass $M_{\Delta}$. The concentration is defined as, $c_{\Delta}=r_{\Delta} / r_{s}$, where $r_{s}$ is a scale radius (denoting the radius at which the logarithmic denstiy slope is -2), and $r_{\Delta}$ is a reference radius defined so that the average density of the halo within the sphere of radius $r_{\Delta}$ equals the number $\Delta$ times the critical density of the Universe. Quoted values for $\Delta$ typically range from 100-2500. The mass, $M_{\Delta}$, is the mass enclosed within $r_{\Delta}$. Often $\Delta$ is chosen to be the solution to the spherical collapse model at the time of cluster virialization (153), where $\Delta \sim 100$ at the present epoch in the standard $\Lambda \mathrm{CDM}$ cosmology, in which case the parameters are sometimes referred to as the "virial radius", $r_{\mathrm{vir}}$, "virial mass", $M_{\mathrm{vir}}$, and "virial concentration", $c_{\mathrm{vir}}$.

Dissipationless simulations of the $\Lambda \mathrm{CDM}$ model predict $c_{\mathrm{vir}} \approx 10$ for DM halos with an intrinsic scatter of $\approx 0.1$ dex over the mass range of elliptical galaxies considered in this review $(155 ;$; 156$)$. Some early measurements obtained much larger concentrations on these mass scales inconsistent with theory. Firstly, (157) attempted to deconvolve the large, energy-dependent PSF of ASCA observations, which is challenging given that the size of the PSF $\left(>1^{\prime}\right)$ is generally as large or larger than the optical half-light radii of nearby elliptical galaxies observed in Xrays. Secondly, (158) used ROSAT observations, but they had to assume the gas is isothermal throughout each galaxy. Finally, one early study using Chandra data of NGC 6482 (159) with an accurately measured (non-isothermal) temperature profile also obtained a very large concentration $\left(c_{200}=61, c_{\text {vir }} \sim 80\right)$.

Mamon \& Lokas (154) proposed that such large concentrations disagreeing with theoretical expectation were primarily an artifact of neglecting to include a component for the stellar mass in X-ray determinations of the DM profile. This bias was confirmed with Chandra observations by (94) in their sample of seven galaxies 

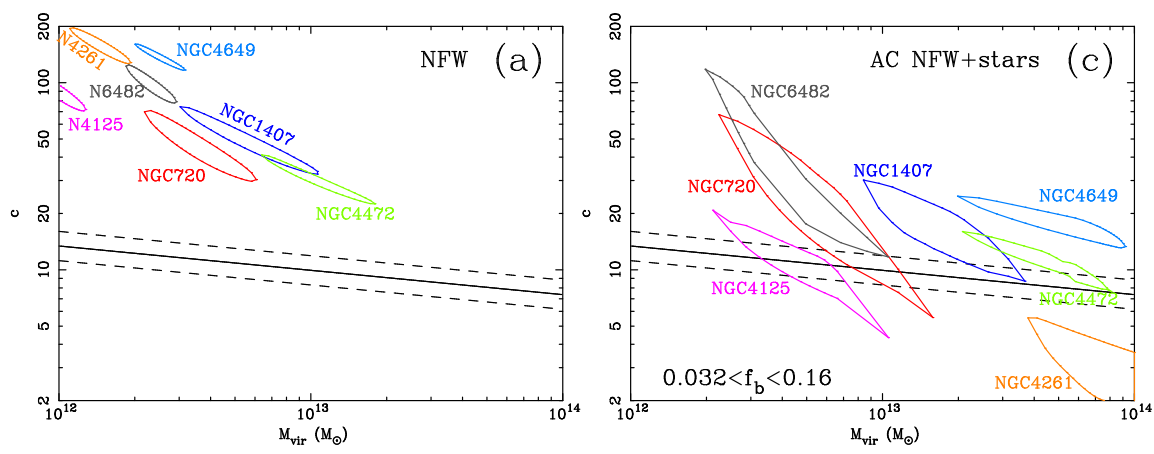

Fig. $6 c_{\text {vir }}-M_{\text {vir }}$ results $(1 \sigma)$ for the seven elliptical galaxies shown in Figure 1 from (94) where the mass model includes (Left Panel) only a single NFW component and (Right Panel) an NFW model for the DM and a Hernquist model for for the stellar mass. The omission of the stellar component leads to a large overestimate of the concentration parameter as originally suggested by (154). When including components for both the stars and DM the values are mostly consistent with the theoretical expectation within the errors (solid line is median and dashed lines $1 \sigma$ intrinsic scatter), with a weak suggestion that the galaxies lie systematically above the theoretical relation.

(Figure 6), demonstrating that reliable X-ray measurements of the DM concentration on the galaxy scale definitely require the stellar mass to be modeled accurately. There is good agreement in the concentrations obtained for studies of galaxies like NGC 1407 that include the stellar mass (94; 146). But statistically significant systematic differences can arise in some (higher mass) galaxies between studies that include stellar mass (65) and those that instead exclude a large portion of the central region (66). As emphasized by (65), since an accurate determination of the DM concentration requires that the NFW scale radius be accurately measured, it is essential that $r_{s}$ lie within the range of data being fitted. One the other hand, excluding the central region of a galaxy disturbed by AGN feedback is a reasonable approach for hydrostatic analysis. In either case, the systematic error in the adopted analysis choice needs to be investigated and quantified.

The observed $c_{\mathrm{vir}}-M_{\mathrm{vir}}$ relation for elliptical galaxies inferred from X-rays (Figure 6) is broadly consistent with the cosmological prediction within the estimated observational errors. Despite the relatively large error regions, these measurements on the elliptical galaxy scale, when combined with measurements for groups and massive clusters, were crucial to providing a sufficiently wide mass baseline to establish that the $c_{\mathrm{vir}}-M_{\mathrm{vir}}$ slope is indeed negative (a robust prediction of CDM models) with a value $(-0.17 \pm 0.03)$ close to the theoretical prediction $(160)$. However, even when accounting for the stellar mass the normalization of the $c_{\mathrm{vir}}-M_{\mathrm{vir}}$ relation on the galaxy scale remains larger than predicted. While the effect is not highly significant for the results shown in Figure 6, the recent analysis of NGC 720 (92) using deeper Chandra data mentioned previously with an improved entropy-based analysis finds $c_{\mathrm{vir}}=26 \pm 5, M_{\mathrm{vir}}=3.3 \pm 0.4 \times 10^{12} M_{\odot}$, which exceeds the theoretical prediction (considering intrinsic scatter) by about $2 \sigma$. The systematic offset could represent one or more of the following: the selection of preferentially early 


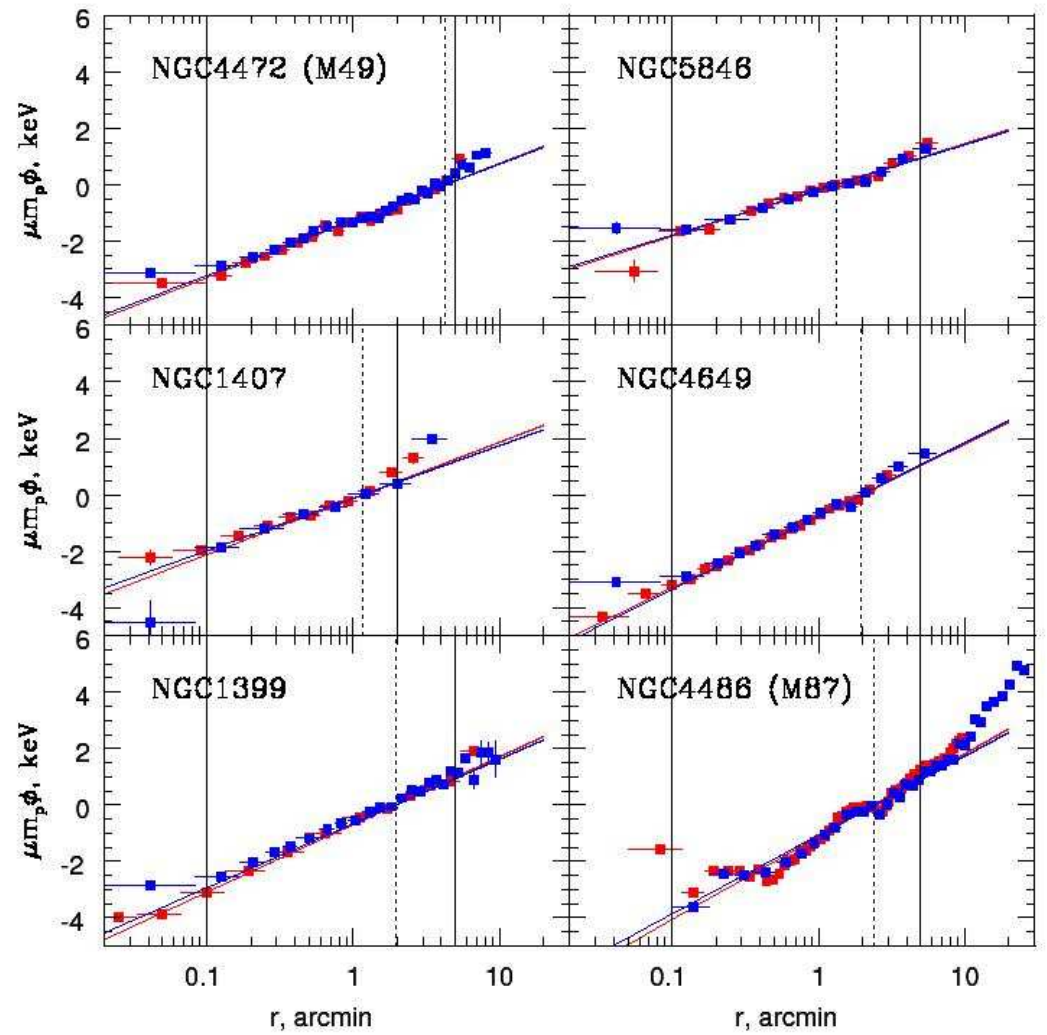

Fig. 7 Gravitational potential profiles and power-law fits from (162). Chandra data are shown in red and $X M M$ in blue. Solid vertical lines indicate the radial range included for the power-law fits, while the vertical dotted lines indicate the optical half-light radius.

forming systems (NGC 720 is a very relaxed isolated galaxy), departures from hydrostatic equilibrium (underestimate of stellar mass contribution), or the need for a different value of $\sigma_{8}$ or $w$ in the cosmological model (see 160). Although adiabatic contraction $(27 ; 161)$ can lower the inferred concentrations, the estimated modest reductions still exceed the theoretical prediction $(94 ; 65 ;$; 92).

While the NFW profile is a good description of the DM profiles in elliptical galaxies, evidence continues to accumulate that the total gravitating mass is very well approximated by a single power-law over a wide radial range. Prior to Chan$d r a$ and $X M M$ many X-ray studies found mass profiles consistent with $\rho \sim r^{-\alpha}$, where $\alpha \approx 2$ (see (162; 163), and references therein), although isothermal gas was 

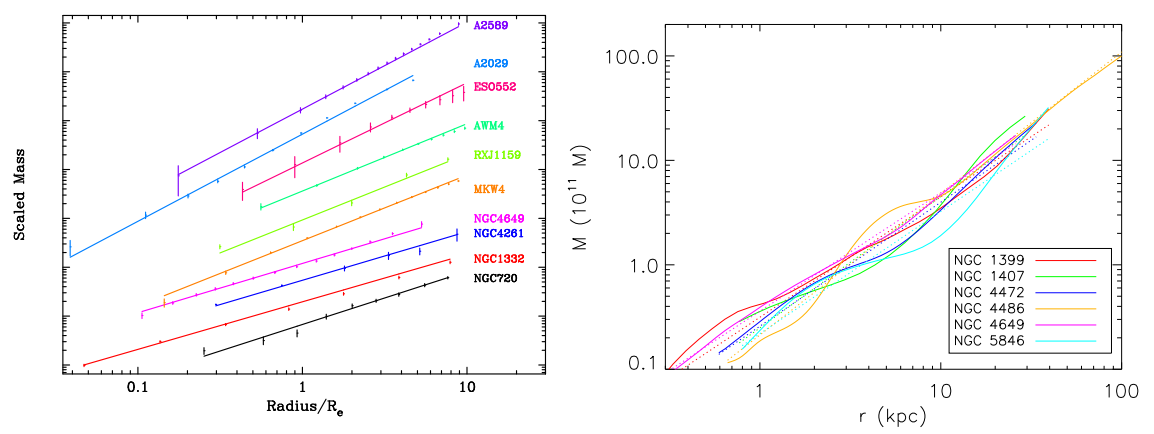

Fig. 8 Radial mass profiles and associated power-law fits. (Left Panel) Results for 10 objects spanning galaxies to clusters from (163), arbitrarily scaled for clarity. The solid lines are the bestfitting profiles determined from an entropy-based forward-fitting procedure, while the data points are determined from the traditional smoothed inversion approach; i.e., the displayed models are not fitted to the data points but are derived independently. (Right Panel) Results for 6 objects from (118) based on X-ray data analyzed by (162). Solid lines show the mass best-fitting mass profiles, while the dotted lines show the best-fitting power-laws to these mass profiles.
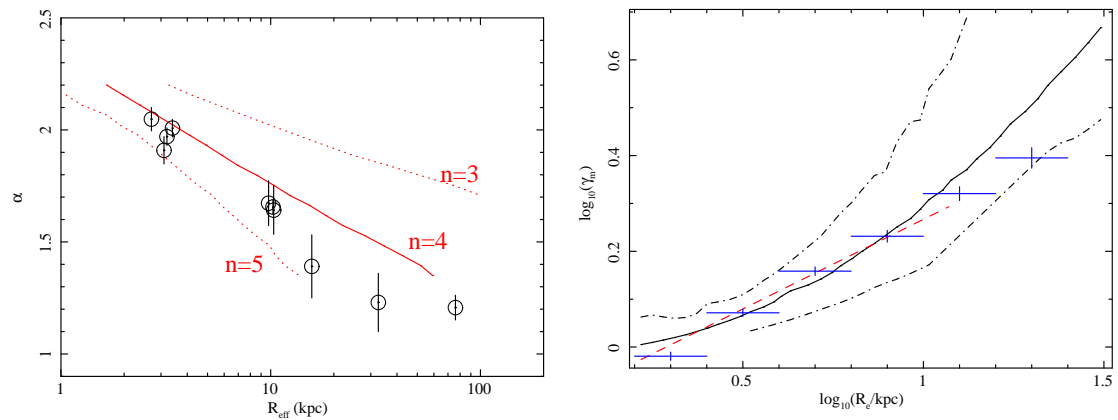

Fig. 9 From (163): (Left Panel) X-ray (black circles) measurements of logarithmic mass slope plotted against optical half-light radius. The red lines are model predictions decomposing a powerlaw total mass profile into Sersic stellar light and NFW DM profiles (see text). (Right Panel) Ratio $\gamma_{m}$ of total mass to stellar mass enclosed within $R_{e}$ plotted vs. $R_{e}$. The solid line is the model prediction corresponding to the $n=4$ curve shown in the left panel which agrees well with the data points corresponding to the $K$-band $M / L$ ratios adapted from the data of (164).

generally assumed. These results have now been confirmed and strengthened (Figures 7. (8) with accurate temperature profiles measured with Chandra and XMM (82; 94; 162; 163; 118). Furthermore, using Chandra data of 10 galaxies spanning a wide range in $\mathrm{M}_{\mathrm{vir}}$ (including some clusters) and optical half-light radii $\left(R_{e}\right),(163)$ discovered that the power-law index $\alpha$ decreases systematically with increasing $R_{e}$ (Figure 9). This behavior can be explained by the combination of the stellar (Sersic) and DM (NFW) profiles required to produce a power-law total mass profile, which leads to the relation $\alpha=2.31-0.54 \log _{10} R_{e}$ for a Sersic index $n \approx 4$ corresponding to a stellar de Vaucouleurs profile. 
This correlation has recently been confirmed by (165) using combined central velocity dispersions and strong gravitational lensing in 73 early-type galaxies. These optical results provide very important supporting evidence, but since they essentially determine a mass profile from two mass data points (i.e., at the center and at the Einstein radius of a galaxy), the X-ray measurements remain of critical importance. This relation between $\alpha$ with $R_{e}$ has far-reaching implications, as (163) showed that it implies a DM fraction within $R_{e}$ that varies systematically with the properties of the galaxy in such a manner as to reproduce, without fine tuning, the observed tilt of the Fundamental Plane. Consequently, (163) speculated that establishing a nearly power-law total mass distribution is a fundamental feature of galaxy formation and the primary factor which determines the tilt of the fundamental plane.

\section{Baryon Fraction}

Please see the full article to be published in the book, Hot Interstellar Matter in Elliptical Galaxies, eds. D.-W. Kim \& S. Pellegrini, Astrophysics \& Space Science Library (ASSL), Springer.

\section{Non-Spherical Constraints}

Please see the full article to be published in the book, Hot Interstellar Matter in Elliptical Galaxies, eds. D.-W. Kim \& S. Pellegrini, Astrophysics \& Space Science Library (ASSL), Springer.

\section{SMBHs}

Please see the full article to be published in the book, Hot Interstellar Matter in Elliptical Galaxies, eds. D.-W. Kim \& S. Pellegrini, Astrophysics \& Space Science Library (ASSL), Springer.

\section{Accuracy of the Hydrostatic Equilibrium Approximation}

Provided other sources of systematic error are controlled, the accuracy of a mass measurement from X-rays reflects the degree to which non-thermal motions are present in the hot gas. As remarked in 2.1 only limited direct constraints on gas motions are possible with current $\mathrm{X}$-ray detectors, and such measurements that exist suggest subsonic non-thermal motions. An indirect assessment of non-thermal mo- 


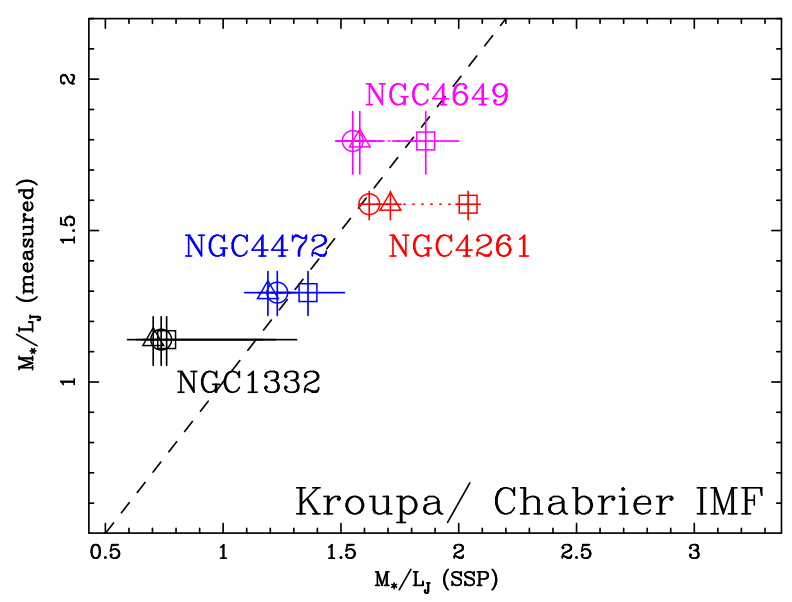

Fig. 10 Comparison of the stellar M/L ratios from X-ray mass modeling with those predicted from three sets of SSP models (Squares: (167); circles: (168); triangles: (169)). The dotted line is " $\mathrm{y}=\mathrm{x}$ ". The good agreement implies deviations from hydrostatic equilibrium are less than $\sim 10$ $20 \%$. Adapted from (91).

tions can be achieved by comparing the X-ray-determined stellar $M / L$ ratios with those predicted for each galaxy by single-burst stellar population synthesis (SSP) models. For a sample of 4 galaxies (including NGC 4472 and NGC 4261, both of which have AGN-blown cavities in the ISM), the X-rays and SSP display good overall agreement (Fig 10, implying non-thermal pressure is no larger than $\sim 20 \%$ (91). Similar agreement was found for NGC $1407(146)$. While the SSP models could be in error due to a mixture of differently aged stars in each galaxy or the initial mass function (IMF) differing from that of Kroupa (166), for non-thermal pressure to be significant, it must exist in a finely balanced conspiracy with the shape of the IMF.

Alternatively, if the three-dimensional gravitational potential can be measured independently, it should be possible to use the measured X-ray properties of the gas to make an (indirect) constraint on non-thermal gas motions. Since elliptical galaxies with X-ray data of sufficient quality for detailed mass analysis are necessarily quite nearby (within $\sim 100 \mathrm{Mpc}$ ), the only techniques (aside from X-ray tools) currently available to measure the gravitational potential with sufficient accuracy are axisymmetric (or triaxial) stellar dynamical methods. The most sophisticated of these methods employ the full information in the line-of-sight stellar velocity distribution to break the mass-orbit degeneracy $(170 ; 171 ; 172$; 173$)$, while making minimal assumptions about the orbital structure $(174 ; 175)$. The fundamentally ill-posed nature of the inversion problem at the heart of these "orbit-based" methods can be mitigated by regularization or a maximum entropy constraint (e.g. 175; 176; 171).

Only a few studies have attempted to combine the X-ray and optical methods in this way. Assuming spherical symmetry and severely limiting the orbital structure by employing "Jeans modeling", a modest disagreement between the mass inferred from the two techniques has been reported for the galaxy NGC 4472 (177; 178). 
While this may indicate on average $\sim 20-50 \%$ non-thermal pressure support, it could also reflect the limitations of Jeans modeling to recover the orbital structure accurately (e.g., 179). In contrast, using a similar approach (180) argued the X-ray method overestimates the mass distribution in the elliptical galaxy NGC 1407 by $\sim 70 \%$, which is difficult to understand unless the gas is globally out-flowing. The exact magnitude of this discrepancy, however, is very uncertain due to large systematic errors associated with assumptions in both the X-ray and the optical analysis. (A similar conclusion obtained for the galaxy NGC 3379 (83) is also very uncertain since unresolved point sources dominate the X-ray emission in that particular galaxy $(84 ;$ 85).) Large systematic uncertainties have also characterized similar studies of 6 galaxies undertaken by (88) and (162), who concluded that, on average, the X-ray data are consistent with modest, but highly uncertain, non-thermal support (see Figure 11). They conclude that, although they find 30\% non-thermal pressure in the systems they studied, "the uncertainties in [their] model assumptions (e.g., spherical symmetry) are sufficiently large that the contribution [of non-thermal pressure] could be consistent with zero." (88). By an extension of this argument, it could also be significantly larger.
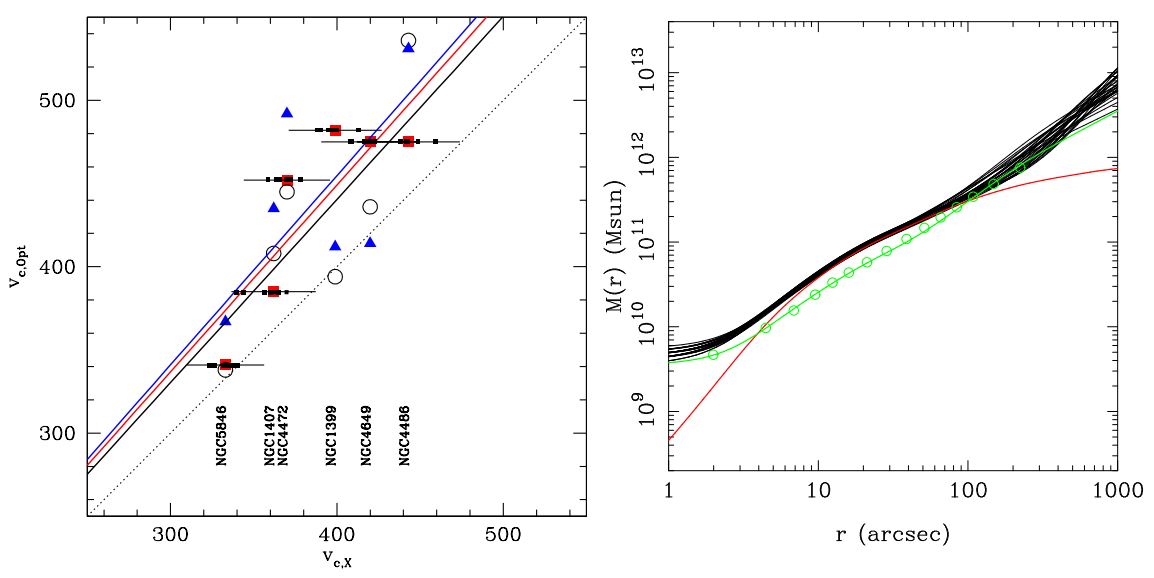

Fig. 11 (Left Panel) Comparison of circular velocities for six nearby galaxies inferred from hydrostatic X-ray models (horizontal axis) and from a suite of simple stellar dynamical estimates (vertical axis). The observation that the galaxies tend to lie above the dotted line representing equal $\mathrm{X}$-ray and optical values implies that the X-ray method tends to underestimate the mass by around $30 \%$, implying significant non-thermal gas motions (162). (Right Panel) Stellar dynamics/X-ray comparison for NGC 4649 from (152). The black lines represent the total mass profile (and $1 \sigma$ range) from the stellar dynamics. The green line is a representation of the mass profile inferred from X-rays (90), and the red line is the stellar mass.

A major roadblock to the use of more rigorous axisymmetric (or triaxial) "orbit based" methods for modeling the optical data in such work has been the pro- 
hibitive computational cost of self-consistently incorporating a DM halo in such a scheme. Recently, however, such models have begun to be developed (e.g., 181). In particular, (152) found a discrepancy between the mass profile of NGC 4649 from orbit-based models and the (spherical) X-ray mass distribution, implying on average $\sim 40 \%$ non-thermal support. This is significantly larger than was required by the hydrodynamical models of (41) to reconcile the stellar and X-ray ellipticity profiles (although this comparison is not strictly self-consistent as the hydrodynamical models assumed a gravitational potential consistent with the X-ray results). Similarly, (173) found discrepancies for the galaxy M 87, while the X-ray mass profiles were found by (118) to underestimate by $\sim 20-45 \%$ the gravitating mass determined from orbit-based stellar models in the centers of three galaxies, with the sign of the discrepancy reversing at large ( $~ 10 \mathrm{kpc}$ ) radii. While inclination effects could conceivably contribute a significant uncertainty into the stellar dynamical analysis of the round galaxies considered in these studies, a similar mismatch between the Xray and (orbit-based) optical mass estimates (corresponding to $\sim 50 \%$ non-thermal support) has been reported in the central $\sim 3 \mathrm{kpc}$ region for the edge-on S0 galaxy NGC 1332 (182).

Hence, the current status of comparisons between stellar dynamics and X-rays is that X-rays tend, on average, to underestimate the mass obtained by stellar dynamics by $\sim 30 \%$, but with considerable uncertainty on many of the measurements, and the comparison can be complicated by a radial dependence in the discrepancy. To better understand the systematic errors in these comparisons, it is essential in the future that self-consistent comparisons be made; i.e., simultaneous modeling of the $\mathrm{X}$-ray and stellar dynamical data. The X-ray data analysis is subject to numerous potential systematic effects which need to be controlled; e.g., background characterization, measurements of the metal abundances, treatment of unresolved discrete sources, and even uncertainties associated with the mass modeling technique that is adopted (e.g., 91; 118). While many of these systematic errors are modest in absolute terms, they can be comparable to, or at times exceed, the statistical errors. With the detailed comparison between X-ray and optical mass profiles now demanding the interpretation of differences as small as $\sim 20 \%$, precise control of these errors is essential. Until this becomes routine, the accurate radial profiles of possible nonthermal gas motions inferred by comparing mass profiles determined from X-rays and stellar dynamics will remain out of reach.

\section{Conclusions}

With the advent of accurate radial temperature profiles from Chandra, XMM, and Suzaku, the need for DM is now firmly established in many giant elliptical galaxies $\left(10^{12} \mathrm{M}_{\odot} \lesssim \mathrm{M}_{\mathrm{vir}} \lesssim 10^{13} \mathrm{M}_{\odot}\right)$. Current data do not distinguish between NFW and isothermal DM profiles. For the cosmologically motivated NFW model the inferred concentration parameters of elliptical galaxies generally exceed the mean theoretical relation. This discrepancy may be due in large part to the preferential selection 
of the most relaxed galaxies for X-ray studies. X-ray observations confirm that the total mass profile (baryons+DM) is close to isothermal $(M \sim r)$, and new evidence suggests a more general power-law relation for the slope of the total mass profile that varies with the stellar half-light radius. While the global baryon fraction remains difficult to constrain, a recent joint Chandra-Suzaku analysis of NGC 720 indicates a global baryon fraction consistent with the cosmological value, suggesting a Milky Way-size galaxy can retain all of its baryons. The axial ratio of the DM inferred from the X-ray isophote shapes of NGC 720 is very consistent with those of halos produced in cosmological simulations. Finally, the unprecedented high spatial resolution of Chandra has enabled the first measurements of the mass of SMBHs in a few elliptical galaxies, obtaining a precision comparable to that achieved by traditional optical methods in NGC 4649.

The most important uncertainty in X-ray determinations of DM in elliptical galaxies is no longer the measurement of the temperature profile but now the accuracy of the hydrostatic equilibrium approximation; i.e., the magnitude of nonthermal motions in the hot ISM. The limited direct constraints possible with current data suggest subsonic non-thermal motions, and improved measurements of turbulent motions via Doppler line broadening in the brightest systems are expected from Astro- $H$ in a few years. In the meantime, it is essential to perform self-consistent studies of X-ray and stellar dynamical data of elliptical galaxies to assess the magnitude of non-thermal gas motions and achieve more reliable DM measurements than possible with either technique individually.

Acknowledgements We thank W. Mathews, F. Brighenti, S. Ettori, and K. Gebhardt for discussions related to this work. We are grateful to W. Mathews and F. Gastaldello for providing comments on the manuscript. We also would like to express our appreciation to E. O'Sullivan for providing data included in Figure 4 This work was supported in part by the National Aeronautics and Space Administration under Grant No. NNX10AD07G issued through the Astrophysics Data Analysis Program (ADP).

\section{References}

1. J.L. Feng, ArXiv High Energy Physics - Phenomenology e-prints (2005)

2. S. Hannestad, International Journal of Modern Physics A 21, 1938 (2006). DOI 10.1142/ S0217751X06032885

3. J. Einasto, ArXiv e-prints (2009)

4. F.D. Steffen, European Physical Journal C 59, 557 (2009). DOI 10.1140/epjc/ s10052-008-0830-0

5. I.G. Irastorza, ArXiv e-prints (2009)

6. J. Ellis, K.A. Olive, ArXiv e-prints (2010)

7. C. Amsler, et al., Phys. Lett. B667, 1 (2008). DOI 10.1016/j.physletb.2008.07.018

8. O. Lahav, A.R. Liddle, ArXiv e-prints (2010)

9. B. Moore, Nature 370, 629 (1994). DOI 10.1038/370629a0

10. R.A. Flores, J.R. Primack, ApJ 427, L1 (1994). DOI 10.1086/187350

11. G. Gilmore, D. Zucker, M. Wilkinson, R.F.G. Wyse, V. Belokurov, J. Kleyna, A. Koch, N.W. Evans, E.K. Grebel, in Astronomical Society of the Pacific Conference Series, Astronomical 
Society of the Pacific Conference Series, vol. 399, ed. by T. Kodama, T. Yamada, \& K. Aoki (2008), Astronomical Society of the Pacific Conference Series, vol. 399, p. 453

12. J.R. Primack, New Journal of Physics 11(10), 105029 (2009). DOI 10.1088/1367-2630/11/ $10 / 105029$

13. W.J.G. de Blok, Advances in Astronomy 2010 (2010). DOI 10.1155/2010/789293

14. D.J. Sand, T. Treu, R.S. Ellis, ApJ 574, L129 (2002). DOI 10.1086/342530

15. D.D. Kelson, A.I. Zabludoff, K.A. Williams, S.C. Trager, J.S. Mulchaey, M. Bolte, ApJ 576, 720 (2002). DOI 10.1086/341891

16. D.J. Sand, T. Treu, G.P. Smith, R.S. Ellis, ApJ 604, 88 (2004). DOI 10.1086/382146

17. D.J. Sand, T. Treu, R.S. Ellis, G.P. Smith, J. Kneib, ApJ 674, 711 (2008). DOI 10.1086/ 524652

18. A.B. Newman, T. Treu, R.S. Ellis, D.J. Sand, J. Richard, P.J. Marshall, P. Capak, S. Miyazaki, ApJ 706, 1078 (2009). DOI 10.1088/0004-637X/706/2/1078

19. J.S. Arabadjis, M.W. Bautz, G.P. Garmire, ApJ 572, 66 (2002). DOI 10.1086/340296

20. A.D. Lewis, D.A. Buote, J.T. Stocke, ApJ 586, 135 (2003)

21. D.N. Spergel, P.J. Steinhardt, Physical Review Letters 84, 3760 (2000). DOI 10.1103/ PhysRevLett.84.3760

22. C.S. Kochanek, M. White, ApJ 543, 514 (2000). DOI 10.1086/317149

23. C. Firmani, E. D’Onghia, G. Chincarini, X. Hernández, V. Avila-Reese, MNRAS 321, 713 (2001). DOI 10.1046/j.1365-8711.2001.04030.x

24. K. Ahn, P.R. Shapiro, MNRAS 363, 1092 (2005). DOI 10.1111/j.1365-2966.2005.09492.x

25. S.W. Randall, M. Markevitch, D. Clowe, A.H. Gonzalez, M. Bradač, ApJ 679, 1173 (2008). DOI 10.1086/587859

26. R. Kuzio de Naray, G.D. Martinez, J.S. Bullock, M. Kaplinghat, ApJ 710, L161 (2010). DOI 10.1088/2041-8205/710/2/L161

27. G.R. Blumenthal, S.M. Faber, J.R. Primack, M.J. Rees, Nature 311, 517 (1984)

28. A.A. El-Zant, Y. Hoffman, J. Primack, F. Combes, I. Shlosman, ApJ 607, L75 (2004). DOI $10.1086 / 421938$

29. O.Y. Gnedin, D.H. Weinberg, J. Pizagno, F. Prada, H. Rix, ApJ 671, 1115 (2007). DOI $10.1086 / 523256$

30. E. Romano-Díaz, I. Shlosman, Y. Hoffman, C. Heller, ApJ 685, L105 (2008). DOI 10.1086/ 592687

31. A. Del Popolo, ApJ 698, 2093 (2009). DOI 10.1088/0004-637X/698/2/2093

32. A.R. Duffy, J. Schaye, S.T. Kay, C. Dalla Vecchia, R.A. Battye, C.M. Booth, MNRAS 405, 2161 (2010). DOI 10.1111/j.1365-2966.2010.16613.x

33. M.G. Abadi, J.F. Navarro, M. Fardal, A. Babul, M. Steinmetz, MNRAS p. 847 (2010). DOI 10.1111/j.1365-2966.2010.16912.x

34. L. Ferrarese, D. Merritt, ApJ 539, L9 (2000). DOI 10.1086/312838

35. K. Gebhardt, R. Bender, G. Bower, A. Dressler, S.M. Faber, A.V. Filippenko, R. Green, C. Grillmair, L.C. Ho, J. Kormendy, T.R. Lauer, J. Magorrian, J. Pinkney, D. Richstone, S. Tremaine, ApJ 539, L13 (2000). DOI 10.1086/312840

36. Y. Birnboim, A. Dekel, MNRAS 345, 349 (2003). DOI 10.1046/j.1365-8711.2003.06955.x

37. D. Kereš, N. Katz, D.H. Weinberg, R. Davé, MNRAS 363, 2 (2005). DOI 10.1111/j. 1365-2966.2005.09451.x

38. Y. Birnboim, A. Dekel, E. Neistein, MNRAS 380, 339 (2007). DOI 10.1111/j.1365-2966. 2007.12074.x

39. W.G. Mathews, F. Brighenti, ARA\&A 41, 191 (2003)

40. A. Dekel, Y. Birnboim, MNRAS 383, 119 (2008). DOI 10.1111/j.1365-2966.2007.12569.x

41. F. Brighenti, W.G. Mathews, P.J. Humphrey, D.A. Buote, ApJ 705, 1672 (2009). DOI 10.1088/0004-637X/705/2/1672

42. T.J. Ponman, D.J. Allan, L.R. Jones, M. Merrifield, I.M. McHardy, H.J. Lehto, G.A. Luppino, Nature 369, 462 (1994). DOI 10.1038/369462a0

43. A. Vikhlinin, B.R. McNamara, A. Hornstrup, H. Quintana, W. Forman, C. Jones, M. Way, ApJ 520, L1 (1999). DOI 10.1086/312134 
44. L.R. Jones, T.J. Ponman, A. Horton, A. Babul, H. Ebeling, D.J. Burke, MNRAS 343, 627 (2003). DOI 10.1046/j.1365-8711.2003.06702.x

45. E. D’Onghia, J. Sommer-Larsen, A.D. Romeo, A. Burkert, K. Pedersen, L. Portinari, J. Rasmussen, ApJ 630, L109 (2005). DOI 10.1086/491651

46. A. Dariush, H.G. Khosroshahi, T.J. Ponman, F. Pearce, S. Raychaudhury, W. Hartley, MNRAS 382, 433 (2007). DOI 10.1111/j.1365-2966.2007.12385.x

47. M. Milosavljević, C.J. Miller, S.R. Furlanetto, A. Cooray, ApJ 637, L9 (2006). DOI 10. $1086 / 500547$

48. G. Fabbiano, ARA\&A 27, 87 (1989). DOI 10.1146/annurev.aa.27.090189.000511

49. D.A. Buote, C.R. Canizares, in Galactic Halos, Astronomical Society of the Pacific Conference Series, vol. 136, ed. by D. Zaritsky (1998), Astronomical Society of the Pacific Conference Series, vol. 136, p. 289

50. O. Gerhard, in Planetary Nebulae Beyond the Milky Way, ed. by L. Stanghellini, J. R. Walsh, \& N. G. Douglas (2006), p. 299. DOI 10.1007/3-540-34270-2-47

51. O. Gerhard, ArXiv e-prints (2010)

52. R.S. Ellis, Phil. Trans. R. Soc. A 368, 967 (2010). DOI 10.1098/rsta.2009.0209

53. T. Treu, ARA\&A 48, 87 (2010). DOI 10.1146/annurev-astro-081309-130924

54. C.L. Sarazin, Reviews of Modern Physics 58, 1 (1986). DOI 10.1103/RevModPhys.58.1

55. A.C. Fabian, in NATO ASIC Proc. 300: Physical Processes in Hot Cosmic Plasmas, ed. by W. Brinkmann, A. C. Fabian, \& F. Giovannelli (1990), pp. 271-297

56. C.L. Sarazin, in NATO ASIC Proc. 366: Clusters and Superclusters of Galaxies, ed. by A. C. Fabian (1992), p. 131

57. R. Mewe, in X-Ray Spectroscopy in Astrophysics, Lecture Notes in Physics, Berlin Springer Verlag, vol. 520, ed. by J. van Paradijs \& J. A. M. Bleeker (1999), Lecture Notes in Physics, Berlin Springer Verlag, vol. 520, p. 109

58. L. Spitzer, Physics of Fully Ionized Gases (1962)

59. L. Spitzer, Jr., ApJ 124, 20 (1956). DOI 10.1086/146200

60. L.L. Cowie, C.F. McKee, ApJ 211, 135 (1977). DOI 10.1086/154911

61. M.A. Dopita, R.S. Sutherland, Astrophysics of the diffuse universe (2003)

62. J.P. Vallée, New Astronomy Review 48, 763 (2004). DOI 10.1016/j.newar.2004.03.017

63. W.G. Mathews, F. Brighenti, ApJ 488, 595 (1997). DOI 10.1086/304728

64. F. Govoni, L. Feretti, International Journal of Modern Physics D 13, 1549 (2004). DOI $10.1142 / \mathrm{S} 0218271804005080$

65. F. Gastaldello, D.A. Buote, P.J. Humphrey, L. Zappacosta, J.S. Bullock, F. Brighenti, W.G. Mathews, ApJ 669, 158 (2007). DOI 10.1086/521519

66. M. Sun, G.M. Voit, M. Donahue, C. Jones, W. Forman, A. Vikhlinin, ApJ 693, 1142 (2009). DOI 10.1088/0004-637X/693/2/1142

67. N. Werner, I. Zhuravleva, E. Churazov, A. Simionescu, S.W. Allen, W. Forman, C. Jones, J.S. Kaastra, MNRAS 398, 23 (2009). DOI 10.1111/j.1365-2966.2009.14860.x

68. J.S. Sanders, A.C. Fabian, R.K. Smith, ArXiv e-prints (2010)

69. J.C. Tsai, N. Katz, E. Bertschinger, ApJ 423, 553 (1994). DOI 10.1086/173834

70. A.E. Evrard, C.A. Metzler, J.F. Navarro, ApJ 469, 494 (1996). DOI 10.1086/177798

71. D. Nagai, A. Vikhlinin, A.V. Kravtsov, ApJ 655, 98 (2007). DOI 10.1086/509868

72. R. Piffaretti, R. Valdarnini, A\&A 491, 71 (2008). DOI 10.1051/0004-6361:200809739

73. T. Fang, P. Humphrey, D. Buote, ApJ 691, 1648 (2009). DOI 10.1088/0004-637X/691/2/ 1648

74. N. Caon, D. Macchetto, M. Pastoriza, ApJS 127, 39 (2000). DOI 10.1086/313315

75. P.C. Hanlan, J.N. Bregman, ApJ 530, 213 (2000). DOI 10.1086/308357

76. R.A. Crain, I.G. McCarthy, C.S. Frenk, T. Theuns, J. Schaye, MNRAS p. 967 (2010). DOI 10.1111/j.1365-2966.2010.16985.x

77. R.A. Crain, I.G. McCarthy, J. Schaye, C.S. Frenk, T. Theuns, ArXiv e-prints (2010)

78. D.A. Buote, C.R. Canizares, ApJ 427, 86 (1994). DOI 10.1086/174123

79. D.A. Buote, C.R. Canizares, ApJ 457, 177 (1996). DOI 10.1086/176721

80. D.A. Buote, J.C. Tsai, ApJ 439, 29 (1995). DOI 10.1086/175148 
81. P.J. Humphrey, D.A. Buote, ApJ 689, 983 (2008). DOI 10.1086/592590

82. Y. Fukazawa, J.G. Botoya-Nonesa, J. Pu, A. Ohto, N. Kawano, ApJ 636, 698 (2006). DOI $10.1086 / 498081$

83. S. Pellegrini, L. Ciotti, MNRAS 370, 1797 (2006). DOI 10.1111/j.1365-2966.2006.10590.x

84. M. Revnivtsev, E. Churazov, S. Sazonov, W. Forman, C. Jones, A\&A 490, 37 (2008). DOI 10.1051/0004-6361:200809889

85. G. Trinchieri, S. Pellegrini, G. Fabbiano, R. Fu, N.J. Brassington, A. Zezas, D. Kim, J. Gallagher, L. Angelini, R.L. Davies, V. Kalogera, A.R. King, S. Zepf, ApJ 688, 1000 (2008). DOI $10.1086 / 592287$

86. W. Forman, C. Jones, E. Churazov, M. Markevitch, P. Nulsen, A. Vikhlinin, M. Begelman, H. Böhringer, J. Eilek, S. Heinz, R. Kraft, F. Owen, M. Pahre, ApJ 665, 1057 (2007). DOI $10.1086 / 519480$

87. E.T. Million, N. Werner, A. Simionescu, S.W. Allen, P.E.J. Nulsen, A.C. Fabian, H. Bohringer, J.S. Sanders, ArXiv e-prints (2010)

88. E. Churazov, W. Forman, A. Vikhlinin, S. Tremaine, O. Gerhard, C. Jones, MNRAS 388, 1062 (2008). DOI 10.1111/j.1365-2966.2008.13507.x

89. K.W. Cavagnolo, B.R. McNamara, P.E.J. Nulsen, C.L. Carilli, C. Jones, L. Birzan, ArXiv e-prints (2010)

90. P.J. Humphrey, D.A. Buote, F. Brighenti, K. Gebhardt, W.G. Mathews, ApJ 683, 161 (2008). DOI 10.1086/589709

91. P.J. Humphrey, D.A. Buote, F. Brighenti, K. Gebhardt, W.G. Mathews, ApJ 703, 1257 (2009). DOI 10.1088/0004-637X/703/2/1257

92. P.J. Humphrey, D.A. Buote, C.R. Canizares, A.C. Fabian, J.M. Miller, ApJ 729, 53 (2011). DOI 10.1088/0004-637X/729/1/53

93. G.W. Pratt, M. Arnaud, R. Piffaretti, H. Böhringer, T.J. Ponman, J.H. Croston, G.M. Voit, S. Borgani, R.G. Bower, A\&A 511, A85 (2010). DOI 10.1051/0004-6361/200913309

94. P.J. Humphrey, D.A. Buote, F. Gastaldello, L. Zappacosta, J.S. Bullock, F. Brighenti, W.G. Mathews, ApJ 646, 899 (2006). DOI 10.1086/505019

95. S. Diehl, T.S. Statler, ApJ 668, 150 (2007). DOI 10.1086/521009

96. C. Jones, W. Forman, A. Vikhlinin, M. Markevitch, L. David, A. Warmflash, S. Murray, P.E.J. Nulsen, ApJ 567, L115 (2002). DOI 10.1086/340114

97. D.A. Buote, A.D. Lewis, F. Brighenti, W.G. Mathews, ApJ 594, 741 (2003)

98. F. Gastaldello, D.A. Buote, P. Temi, F. Brighenti, W.G. Mathews, S. Ettori, ApJ 693, 43 (2009). DOI 10.1088/0004-637X/693/1/43

99. L.P. David, C. Jones, W. Forman, P. Nulsen, J. Vrtilek, E. O'Sullivan, S. Giacintucci, S. Raychaudhury, ApJ 705, 624 (2009). DOI 10.1088/0004-637X/705/1/624

100. L.P. David, C. Jones, W. Forman, S. Daines, ApJ 428, 544 (1994). DOI 10.1086/174264

101. D.A. Buote, F. Brighenti, W.G. Mathews, ApJ 607, L91 (2004)

102. D.A. Buote, ApJ 574, L135 (2002). DOI 10.1086/342532

103. D.A. Buote, A.D. Lewis, F. Brighenti, W.G. Mathews, ApJ 595, 151 (2003)

104. D. Kim, G. Fabbiano, ApJ 613, 933 (2004). DOI 10.1086/423266

105. P.J. Humphrey, D.A. Buote, ApJ 639, 136 (2006). DOI 10.1086/499323

106. K. Matsushita, Y. Fukazawa, J.P. Hughes, T. Kitaguchi, K. Makishima, K. Nakazawa, T. Ohashi, N. Ota, T. Tamura, M. Tozuka, T.G. Tsuru, Y. Urata, N.Y. Yamasaki, PASJ 59, 327 (2007)

107. J. Rasmussen, T.J. Ponman, MNRAS 380, 1554 (2007). DOI 10.1111/j.1365-2966.2007. 12191.x

108. M. Komiyama, K. Sato, R. Nagino, T. Ohashi, K. Matsushita, PASJ 61, 337 (2009)

109. A.C. Fabian, E.M. Hu, L.L. Cowie, J. Grindlay, ApJ 248, 47 (1981). DOI 10.1086/159128

110. G.A. Kriss, D.F. Cioffi, C.R. Canizares, ApJ 272, 439 (1983). DOI 10.1086/161311

111. P.E.J. Nulsen, H. Bohringer, MNRAS 274, 1093 (1995)

112. D.A. Buote, ApJ 539, 172 (2000). DOI 10.1086/309224

113. W.G. Mathews, ApJ 219, 413 (1978). DOI 10.1086/155794

114. D. Fabricant, M. Lecar, P. Gorenstein, ApJ 241, 552 (1980). DOI 10.1086/158369 
115. L.P. David, P.E.J. Nulsen, B.R. McNamara, W. Forman, C. Jones, T. Ponman, B. Robertson, M. Wise, ApJ 557, 546 (2001). DOI 10.1086/322250

116. L.M. Voigt, A.C. Fabian, MNRAS 368, 518 (2006). DOI 10.1111/j.1365-2966.2006.10199.x

117. P.E.J. Nulsen, S.L. Powell, A. Vikhlinin, ArXiv e-prints (2010)

118. P. Das, O. Gerhard, E. Churazov, I. Zhuravleva, ArXiv e-prints (2010)

119. D. Merritt, B. Tremblay, AJ 108, 514 (1994). DOI 10.1086/117088

120. J. Magorrian, MNRAS 302, 530 (1999). DOI 10.1046/j.1365-8711.1999.02135.x

121. A. Cavaliere, R. Fusco-Femiano, A\&A 49, 137 (1976)

122. C.L. Sarazin, J.N. Bahcall, ApJS 34, 451 (1977). DOI 10.1086/190457

123. A. Cavaliere, R. Fusco-Femiano, A\&A 70, 677 (1978)

124. D.A. White, C. Jones, W. Forman, MNRAS 292, 419 (1997)

125. S.W. Allen, S. Ettori, A.C. Fabian, MNRAS 324, 877 (2001). DOI 10.1046/j.1365-8711. 2001.04318.x

126. A. Cavaliere, A. Lapi, R. Fusco-Femiano, ApJ 698, 580 (2009). DOI 10.1088/0004-637X/ $698 / 1 / 580$

127. R. Fusco-Femiano, A. Cavaliere, A. Lapi, ApJ 705, 1019 (2009). DOI 10.1088/0004-637X/ 705/1/1019

128. A.C. Fabian, P.A. Thomas, R.E. White, III, S.M. Fall, MNRAS 221, 1049 (1986)

129. S.A. Balbus, ApJ 562, 909 (2001). DOI 10.1086/323875

130. E. Quataert, ApJ 673, 758 (2008). DOI 10.1086/525248

131. I.J. Parrish, E. Quataert, P. Sharma, ApJ 712, L194 (2010). DOI 10.1088/2041-8205/712/2/ L194

132. M. Ruszkowski, S.P. Oh, ApJ 713, 1332 (2010). DOI 10.1088/0004-637X/713/2/1332

133. P. Tozzi, C. Norman, ApJ 546, 63 (2001). DOI 10.1086/318237

134. G.M. Voit, S.T. Kay, G.L. Bryan, MNRAS 364, 909 (2005). DOI 10.1111/j.1365-2966.2005. 09621.x

135. E. O'Sullivan, T.J. Ponman, MNRAS 354, 935 (2004). DOI 10.1111/j.1365-2966.2004. 08257.x

136. E. O'Sullivan, A.J.R. Sanderson, T.J. Ponman, MNRAS 380, 1409 (2007). DOI 10.1111/j. 1365-2966.2007.12229.x

137. R. Nagino, K. Matsushita, A\&A 501, 157 (2009). DOI 10.1051/0004-6361/200810978

138. D.A. Buote, C.R. Canizares, ApJ 468, 184 (1996). DOI 10.1086/177680

139. D.A. Buote, C.R. Canizares, ApJ 474, 650 (1997). DOI 10.1086/303490

140. D.A. Buote, T.E. Jeltema, C.R. Canizares, G.P. Garmire, ApJ 577, 183 (2002). DOI 10.1086/ 342158

141. C. Jones, C. Stern, W. Forman, J. Breen, L. David, W. Tucker, M. Franx, ApJ 482, 143 (1997). DOI 10.1086/304104

142. J.A. Irwin, C.L. Sarazin, ApJ 471, 683 (1996)

143. F. Brighenti, W.G. Mathews, ApJ 486, L83 (1997)

144. M. Sun, W. Forman, A. Vikhlinin, A. Hornstrup, C. Jones, S.S. Murray, ApJ 598, 250 (2003). DOI $10.1086 / 378887$

145. E. O’Sullivan, J.M. Vrtilek, D.E. Harris, T.J. Ponman, ApJ 658, 299 (2007). DOI 10.1086/ 511778

146. Z. Zhang, H. Xu, Y. Wang, T. An, Y. Xu, X. Wu, ApJ 656, 805 (2007). DOI 10.1086/510281

147. H. Hoekstra, B.C. Hsieh, H.K.C. Yee, H. Lin, M.D. Gladders, ApJ 635, 73 (2005). DOI $10.1086 / 496913$

148. M. Kleinheinrich, P. Schneider, H. Rix, T. Erben, C. Wolf, M. Schirmer, K. Meisenheimer, A. Borch, S. Dye, Z. Kovacs, L. Wisotzki, A\&A 455, 441 (2006). DOI 10.1051/0004-6361: 20042606

149. R. Mandelbaum, U. Seljak, G. Kauffmann, C.M. Hirata, J. Brinkmann, MNRAS 368, 715 (2006). DOI 10.1111/j.1365-2966.2006.10156.x

150. C. Heymans, E.F. Bell, H. Rix, M. Barden, A. Borch, J.A.R. Caldwell, D.H. McIntosh, K. Meisenheimer, C.Y. Peng, C. Wolf, S.V.W. Beckwith, B. Häußler, K. Jahnke, S. Jogee, S.F. Sánchez, R. Somerville, L. Wisotzki, MNRAS 371, L60 (2006). DOI 10.1111/j.1745-3933. 
2006.00208.x

151. F. Prada, M. Vitvitska, A. Klypin, J.A. Holtzman, D.J. Schlegel, E.K. Grebel, H. Rix, J. Brinkmann, T.A. McKay, I. Csabai, ApJ 598, 260 (2003). DOI 10.1086/378669

152. J. Shen, K. Gebhardt, ApJ 711, 484 (2010). DOI 10.1088/0004-637X/711/1/484

153. G.L. Bryan, M.L. Norman, ApJ 495, 80 (1998). DOI 10.1086/305262

154. G.A. Mamon, E.L. Łokas, MNRAS 362, 95 (2005). DOI 10.1111/j.1365-2966.2005.09225.x

155. J.S. Bullock, T.S. Kolatt, Y. Sigad, R.S. Somerville, A.V. Kravtsov, A.A. Klypin, J.R. Primack, A. Dekel, MNRAS 321, 559 (2001). DOI 10.1046/j.1365-8711.2001.04068.x

156. A.V. Macciò, A.A. Dutton, F.C. van den Bosch, MNRAS 391, 1940 (2008). DOI 10.1111/j. 1365-2966.2008.14029.x

157. S. Sato, F. Akimoto, A. Furuzawa, Y. Tawara, M. Watanabe, Y. Kumai, ApJ 537, L73 (2000). DOI $10.1086 / 312772$

158. X. Wu, Y. Xue, ApJ 529, L5 (2000). DOI 10.1086/312451

159. H.G. Khosroshahi, L.R. Jones, T.J. Ponman, MNRAS 349, 1240 (2004). DOI 10.1111/j. 1365-2966.2004.07575.x

160. D.A. Buote, F. Gastaldello, P.J. Humphrey, L. Zappacosta, J.S. Bullock, F. Brighenti, W.G. Mathews, ApJ 664, 123 (2007). DOI 10.1086/518684

161. O.Y. Gnedin, A.V. Kravtsov, A.A. Klypin, D. Nagai, ApJ 616, 16 (2004)

162. E. Churazov, S. Tremaine, W. Forman, O. Gerhard, P. Das, A. Vikhlinin, C. Jones, H. Böhringer, K. Gebhardt, MNRAS 404, 1165 (2010). DOI 10.1111/j.1365-2966.2010. 16377.x

163. P.J. Humphrey, D.A. Buote, MNRAS 403, 2143 (2010). DOI 10.1111/j.1365-2966.2010. 16257.x

164. F. La Barbera, G. Busarello, P. Merluzzi, I.G. de la Rosa, G. Coppola, C.P. Haines, ApJ 689, 913 (2008). DOI 10.1086/592769

165. M.W. Auger, T. Treu, A.S. Bolton, R. Gavazzi, L.V.E. Koopmans, P.J. Marshall, L.A. Moustakas, S. Burles, ArXiv e-prints (2010)

166. P. Kroupa, MNRAS 322, 231 (2001). DOI 10.1046/j.1365-8711.2001.04022.x

167. C. Maraston, MNRAS 362, 799 (2005). DOI 10.1111/j.1365-2966.2005.09270.x

168. M. Fioc, B. Rocca-Volmerange, A\&A 326, 950 (1997)

169. G. Bruzual, S. Charlot, MNRAS 344, 1000 (2003). DOI 10.1046/j.1365-8711.2003.06897.x

170. R.P. van der Marel, M. Franx, ApJ 407, 525 (1993). DOI 10.1086/172534

171. K. Gebhardt, D. Richstone, J. Kormendy, T.R. Lauer, E.A. Ajhar, R. Bender, A. Dressler, S.M. Faber, C. Grillmair, J. Magorrian, S. Tremaine, AJ 119, 1157 (2000). DOI 10.1086/ 301240

172. R.C.E. van den Bosch, G. van de Ven, E.K. Verolme, M. Cappellari, P.T. de Zeeuw, MNRAS 385, 647 (2008). DOI 10.1111/j.1365-2966.2008.12874.x

173. K. Gebhardt, J. Thomas, ApJ 700, 1690 (2009). DOI 10.1088/0004-637X/700/2/1690

174. M. Schwarzschild, ApJ 232, 236 (1979). DOI 10.1086/157282

175. R.P. van der Marel, N. Cretton, P.T. de Zeeuw, H. Rix, ApJ 493, 613 (1998). DOI 10.1086/ 305147

176. D.O. Richstone, S. Tremaine, ApJ 327, 82 (1988). DOI 10.1086/166171

177. W.G. Mathews, F. Brighenti, ApJ 599, 992 (2003). DOI 10.1086/379537

178. L. Ciotti, S. Pellegrini, MNRAS 350, 609 (2004). DOI 10.1111/j.1365-2966.2004.07670.x

179. J.J. Binney, R.L. Davies, G.D. Illingworth, ApJ 361, 78 (1990). DOI 10.1086/169169

180. A.J. Romanowsky, J. Strader, L.R. Spitler, R. Johnson, J.P. Brodie, D.A. Forbes, T. Ponman, AJ 137, 4956 (2009). DOI 10.1088/0004-6256/137/6/4956

181. J. Thomas, R.P. Saglia, R. Bender, D. Thomas, K. Gebhardt, J. Magorrian, E.M. Corsini, G. Wegner, MNRAS 382, 657 (2007). DOI 10.1111/j.1365-2966.2007.12434.x

182. S.P. Rusli, J. Thomas, P. Erwin, R.P. Saglia, N. Nowak, R. Bender, ArXiv e-prints (2010) 